\title{
Entre programme et processus : le dynamisme de l'écriture flaubertienne \\ Quelques points de méthode
}

\author{
Stéphanie Dord-Crouslé
}

\section{$\mathrm{D}$}

E Madame Bovary À Bouvard ET PÉCuCHet, les dossiers de plans et scénarios flaubertiens commencent à être bien connus : ils ont fait l'objet d'éditions à caractère génétique, éditions dont la nature est cependant fort diverse ${ }^{1}$. La documentation exogénétique, elle aussi, suscite l'intérêt des chercheurs, l'édition des Carnets de travail suffit à le prouver ${ }^{2}$. En revanche, l'immense chantier des brouillons restait, il y a quelques années encore, une terra incognita qui n'avait vu s'aventurer sur ses marges que quelques pionniers. Ces incursions avaient toujours pour but des prélèvements spécifiques effectués dans l'ensemble des brouillons, et strictement délimités à partir du texte définitif de l'œuvre 3 . Un pas décisif a été franchi avec l'apparition de projets de plus large envergure, et l'édition intégrale de corpus ${ }^{4}$. Néanmoins, du fait de l'insuffisante prise en compte de la spécificité de leur matériau et, souvent, de la reconduction de méthodes philologiques inadaptées, ces entreprises pèchent par un défaut de théorisation de la matière qu'elles s'attachent à décrire et à organiser. Aussi les tentatives méthodologiques et typologiques se multiplient-elles maintenant.

Dans un article récent 5 , Pierre-Marc de Biasi définit la strate des brouillons comme une entité hétérogène :

Elle couvre [...] l'intégralité de la phase rédactionnelle qui a occupé l'écrivain à peu près sans interruption pendant plus de quatre années. En réalité, pour l'écriture flaubertienne, la notion typologique de «brouillon» n'est pas suffisamment discriminante pour définir l'unité d'une strate. Pour ce que l'on en sait actuellement, les «brouillons » de Madame Bovary semblent eux-mêmes stratifiés en plusieurs couches rédactionnelles où l'on trouve, semble-t-il, la strate des scénarios développés et des ébauches d'ensemble, puis, la structure de l'œuvre se précisant, les strates de brouillon proprement dites (qui progressent partie par partie, mouvement par mouvement, secteur par secteur), et enfin la strate des mises au net corrigées et celle du manuscrit : soit au total une bonne quarantaine de strates rédactionnelles. 6

Le processus rédactionnel de Flaubert est en effet intimement travaillé par l'hétérogénéité et la prolifération :

1. La dernière en date est celle d'Yvan Leclerc, Plans et scénarios de "Madame Bovary», présentation, transcription et notes par Yvan Leclerc, collection Manuscrits, Zulma - CNRS Éditions, 1995, 199 p. (Voir icimême, p. 53-62, la lecture que Raymonde Debray Genette donne de ce dossier.) On peut aussi citer Alberto Cento, Édition critique de «Bouvard et Pécuchet », précédée des scénarios inédits, Naples, Istituto universitario orientale, et Paris, Nizet, 1964, 597 p. - Giovanni Bonaccorso, Un Cœur simple, «Corpus Flaubertianum I», édition diplomatique et génétique des manuscrits, Les Belles-Lettres, 1983, 586 p.; et Hérodias, «Corpus Flaubertianum II », tome 1, Nizet, 1991, 398 p. ; tome 2, Sicania, 1995, 481 p. - Tony Williams, «L'Éducation sentimentale» - les scénarios, José Corti, 1992, 378 p.

2. Gustave Flaubert, Carnets de travail, édition critique et génétique établie par P.-M. de Biasi, Balland, 1988, 1000 p.

3. Par exemple, l'élaboration progressive d'une scène importante comme la fin d'Un Cæur simple (Raymonde Debray Genette : "Comment faire une fin », repris dans Métamorphoses du récit, Seuil, 1988, p. 85-112) ou de L'Éducation sentimentale (Peter Michael Wetherill : «C'est là ce que nous avons eu de meilleur ", in Flaubert à l'œuvre (Raymonde Debray Genette éd.), Flammarion, 1980, p. 35-68).

4. On citera Giovanni Bonaccorso (pour les travaux détaillés dans la note 1) et Pierre-Marc de Biasi : Édition critique et génétique de «La Légende de saint Julien l'Hospitalier» de G. Flaubert, Histoire, classement, transcription, interprétation des documents de rédaction, texte de l'œuvre, notes, étude de genèse $(5$ vol., dactylog., 940 p.; thèse de doctorat de $3 \mathrm{e}$ cycle en sémiologie, Université Paris VII, 1982).

5. Pierre-Marc de Biasi, «Édition horizontale, édition verticale. Pour une typologie des éditions génétiques (le domaine français 1980-1995)», Éditer des manuscrits - complétude, lisibilité, Saint-Denis, PUV, 1996, p. 159-193.

6. Ibid., p. 171. P.-M. de Biasi se réfère ici aux travaux en cours de M. Durel. 
prolifération des réécritures successives; hétérogénéité des strates entre elles (certaines d'orientation syntagmatique, d'autres d'orientation paradigmatique, en interaction les unes avec les autres) et hétérogénéité typologique des folios à l'intérieur même de chaque strate. Or pour rendre compte efficacement de ces phénomènes, il faut isoler différentes phases récurrentes au sein du processus, et comprendre leur mode d'articulation, c'est-à-dire le rythme de l'écriture. Une fois cette étape franchie, on pourra mettre en valeur le dynamisme de l'écriture propre à l'endogenèse flaubertienne.

\section{Écriture à processus /écriture à programme}

Cherchant à caractériser l'écriture de Flaubert, on s'est tourné vers la distinction opérée par Louis Hay entre l'écriture à programme et l'écriture à processus 7 . Rappelons brièvement en quoi elle consiste.

L'écriture à processus donne d'emblée une version narrativisée de l'œuvre en devenir. Sa «substance est faite d'un travail constant d'écriture ${ }^{8}$. C'est donc un «type d'écriture sans phase préparatoire, sans plan, toujours déjà textualisant $» 9$. Proust a été donné comme l'exemple même de l'écriture à processus ${ }^{10}$. Dans ses brouillons, on ne trouve pas de plan qui connaîtrait un développement ultérieur circonstancié.

Selon nous, on peut aller plus loin en distinguant deux types d'écriture à processus : celle qui parvient à l'œuvre définitive d'une seule «coulée», et celle qui doit recourir à plusieurs versions successives, versions qui n'entretiennent aucun rapport d'ordre paradigmatique entre elles, sinon d'avoir appartenu en fin de compte à la gestation d'une seule et même œuvre.

La figure 1 propose une modélisation de ces deux types d'écriture à processus. Le premier symbolise le déroulement uniforme du texte, de son début à sa fin, son enrichissement continu sous forme textuelle. En revanche, le deuxième type met en valeur les versions successives que peut connaître une même œuvre. Chaque version se constitue suivant le mode décrit pour le premier type. Au nombre de quatre dans le cas fictif choisi, elles ne dépendent pas d'un programme commun, mais élaborent, dans un temps de genèse qui leur est propre, une réalisation possible de l'œuvre. Au terme de leur rédaction, les trois premières ayant été successivement déclarées insatisfaisantes par l'écrivain, la quatrième prend le statut d'œuvre finale11.

L'écriture à programme, au contraire, est un «type d'écriture qui obéit à un programme préétabli et dont l'élaboration parcourt plusieurs états génétiques (notes documentaires, plans, listes, esquisses, brouillons) ${ }^{12}$. $\gg$ Zola est l'écrivain qui se prête le plus naturellement à l'illustration d'un tel procédé, comme le montre Henri Mitterand13 :

[...] Zola offre un excellent exemple de genèse méthodique, définissant explicitement ses objectifs, son programme didactique et narratif, rationalisant de manière quasi obsessionnelle l'ordre et les mesures de son dossier préparatoire, multipliant les contrôles contre toute éventuelle "panne» thématique ou technique, imposant à la fiction la finalité d'une stratégie.

Chaque élément du scénario est successivement développé, et l'ordre d'élaboration est invariant : on va toujours du scénario vers le brouillon sans qu'il y ait jamais aucun phénomène d'échange en sens inverse. La programmation est d'emblée complète, du moins lorsque l'écrivain se lance dans la rédaction d'un chapitre particulier. Car au niveau du roman entier, l'attitude programmatique est plus complexe.

Comme le souligne Colette Becker ${ }^{14}$, l'écriture de «l'Ébauche» est suivie d'une intense recherche documen-

7. Louis Hay, «Nouvelles notes de critique génétique : la troisième dimension de la littérature», Texte, 5-6, 1986-1987, p. 313-328.

8. Louis Hay, ibid., p. 320.

9. Almuth Grésillon, Éléments de critique génétique, PUF, 1994, p. 243 («Glossaire de critique génétique »).

10. Voir Almuth Grésillon, «Proust ou l'écriture vagabonde», in Marcel Proust - Écrire sans fin, textes réunis par R. Warning et J. Milly, CNRS Éditions, 1996, p. 99-124.

11. Ainsi, la nouvelle Blickwechsel est la première version (génétiquement dépendante mais narrativement autonome) de Kindheitsmuster. Voir à ce propos l'article de Louis Hay, «Les trente-trois débuts de Christa Wolf », in Genèses du roman contemporain - Incipit et entrée en écriture, textes réunis par Bernhild Boie et Daniel Ferrer, collection «Textes et manuscrits », CNRS Éditions, 1993, p. 79-102.

12. Almuth Grésillon, Éléments..., p. 243.

13. Henri Mitterand, «Programmes et préconstruits génétiques : le dossier de L'Assommoir», in Essais de critique génétique, Flammarion, 1979, p. 210.

14. Colette Becker, Émile Zola, La fabrique de Germinal, SEDES et Presses Universitaires de Lille, 1986, p. 23 à 25. 

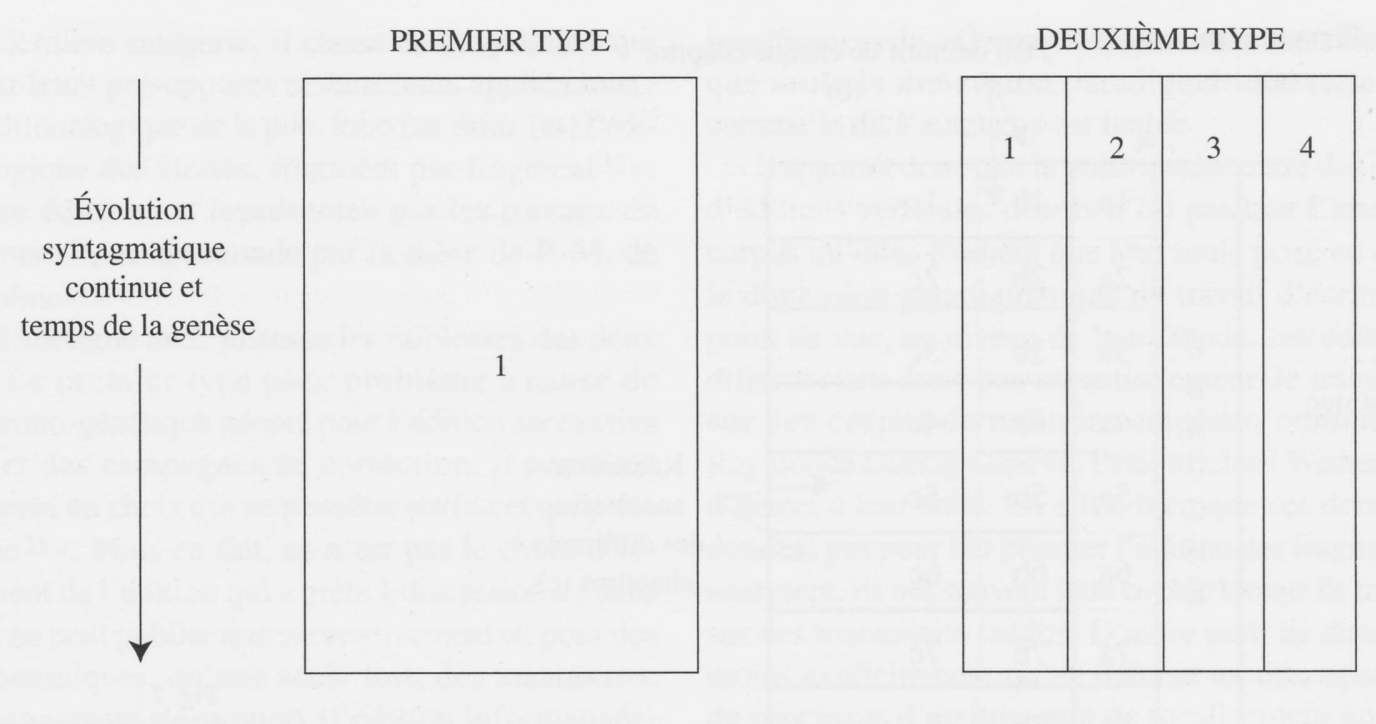

Fig. 1 : L'écriture à processus

taire, puis de l'élaboration d'un «premier plan» de l'œuvre, divisée en chapitres dans lesquels toute la matière rassemblée auparavant doit entrer. Mais la rédaction de chaque chapitre est précédée d'un nouveau plan, plus précis, appelé «plan définitif», et dans lequel peuvent apparaitre des faits directement issus d'un développement rédactionnel précédent, et qui n'avaient pas été prévus par le premier plan de l'œuvre. Ce constat pose donc l'existence d'une circulation possible entre les brouillons textualisants et le scénario des chapitres suivants. Mais cette interaction typologiquement régressive (puisque un brouillon modifie du scénario) semble se cantonner au niveau précédant la phase de textualisation du chapitre. Une fois lancée, la rédaction a en revanche besoin d'un terrain suffisamment solide sous ses pas, qui n'autorise plus de brusque revirement dans sa ligne générale, ni de soudain développement auparavant imprévu 15 .

Dans la figure 2, le temps de la genèse ne se confond plus (comme dans la figure 1) avec un enchaînement syntagmatique continu. Il est plus complexe et voit alterner des phases d'orientation syntagmatique (l'ébauche et le premier plan) et des moments où le processus se fait paradigmatique (l'élaboration de chaque plan définitif précède direc- tement la rédaction du chapitre qu'il concerne). La genèse d'une œuvre zolienne est donc constituée successivement par l'ébauche, le premier plan (1a à 8a), le plan définitif du premier chapitre (1b) et la rédaction de ce dernier (1c), le plan définitif du second chapitre $(2 b)$ et la rédaction de ce dernier (2c), etc.

Mais si la distinction entre écriture à processus et écriture à programme présente un caractère opératoire et des vertus éclairantes non négligeables, on constate cependant qu'elle tend à s'abolir dans la pratique : l'effectuation du programme est sous-tendue par un processus, et le processus dépend d'un programme plus ou moins clairement

15. Néanmoins, on possède peu de brouillons zoliens...

16. Voir Daniel Ferrer, «La toque de Clementis - Rétroaction et rémanence dans les processus génétiques », Genesis 6, 1994, p. 93-106. L'auteur met bien en valeur la ductilité de l'opposition. D'un côté, «l'œuvre [...] implique nécessairement un projet (à l'égard de cette nécessité, [...] l'écriture à processus apparaît comme un cas particulier, une forme minimale, de l'écriture à programme)» (p. 94); mais d'un autre côté, «l'intention se construit chemin faisant, au cours de l'élaboration de l'œuvre (c'est, de ce point de vue, l'écriture à programme qui devient un cas particulier d'écriture à processus) et la situer à l'origine relève d'un mythe commode » (p. 98). 


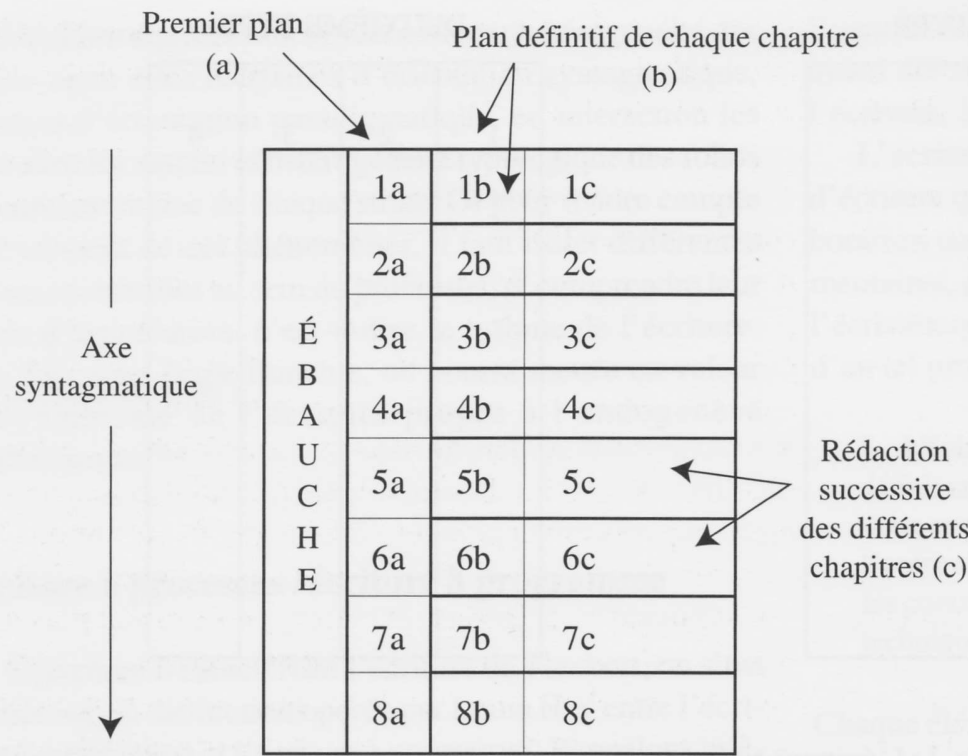

Fig. 2 :

L'écriture à programme (statique) défini, même s'il n'a pas laissé de traces écrites ${ }^{16}$. En outre, la seule notion d'écriture à programme ne rend pas pleinement compte de la pratique de Flaubert « dont les dossiers génétiques témoignent incontestablement d'un esprit planificateur, mais qu'une inventio toujours en éveil conduit tant de fois ailleurs 17 ». Du reste, alors même qu'il posait sa distinction, Louis Hay affirmait déjà «qu'entre les deux grands pôles de l'écriture - processus et programme - se rencontrent un certain nombre de formes intermédiaires ${ }^{18}$ ». C'est justement l'une de ces formes intermédiaires que l'on voudrait isoler, celle qui est propre à l'écriture flaubertienne, apportant ainsi une contribution à la « description typologique des processus de genèse 19 » que Louis Hay appelait de ses vœux. Car, si l'écriture de Flaubert tient plus du programme que du processus, ce programme présente toutefois la particularité d'être sans cesse et méthodiquement réinformé à partir de l'élaboration rédactionnelle qui précède.

\section{Genèse de l'ensemble clos/genèse fragmentale}

Mais avant de procéder à cette définition, il est nécessaire d'en délimiter le cadre théorique. En effet, depuis l'apparition des études de genèse, les analyses de corpus flaubertiens se sont succédé sans que leurs méthodes, pour le moins dissemblables, aient fait véritablement l'objet d'une réflexion d'ensemble. Ce n'est que depuis peu que l'on tente de clarifier et de comprendre la diversité de ces expériences. Les éléments avancés par Pierre-Marc de Biasi20 en vue de la constitution d'une typologie des éditions génétiques fourniront donc le point de départ de cette réflexion.

\section{Hégémonie de l'orientation paradigmatique}

Pierre-Marc de Biasi opère une distinction entre édition horizontale et édition verticale ${ }^{21}$. Au sein de cette dernière, il sépare les éditions partielles des éditions intégrales. Enfin,

17. Almuth Grésillon, Éléments..., p. 104.

18. Louis Hay, op. cit., p. 320.

19. Ibid.

20. Dans son article déjà cité : «Édition horizontale...».

21 . «[...] toutes les éditions génétiques semblent pouvoir se répartir en deux ensembles fonctionnels correspondant à deux grandes orientations éditoriales : les éditions horizontales consacrées à un moment précis du processus de genèse et les éditions verticales visant la reconstitution plus ou moins intégrale de ce processus», (p. 165). 
dans cette dernière catégorie, il classe deux systèmes qui diffèrent par leurs présupposés et dans leurs applications : «l'édition chronologique de la pile, folio par folio, [et] l'édition téléologique des strates, fragment par fragment 22 ». La première édition est représentée par les travaux de G. Bonaccorso 23 , et la seconde par la thèse de P.-M. de Biasi lui-même 24 .

Celui-ci souligne avec justesse les faiblesses des deux systèmes. Le premier type pose problème à cause de «l'ordre chrono-génétique adopté pour l'édition successive des folios et des campagnes de correction, il constitue nécessairement un choix qui ne peut être parfait et qui prête à discussion 25 ». Mais en fait, ce n'est pas le choix d'ordonnancement de l'édition qui «prête à discussion » : dans un livre, on ne peut publier que successivement et, pour des raisons économiques, qu'une seule fois, des manuscrits. Seul un changement de support (l'édition informatisée) pourrait transformer radicalement ces données. Dans le cadre de l'imprimé, le problème vient de ce que le système de classement proposé par G. Bonaccorso n'est que la répétition de l'ordre dans lequel les folios sont déjà donnés dans l'édition. Table des contenus et classement chronologique se confondent. Le second n'apporte rien de plus que le premier, alors qu'on attendrait d'un tel classement qu'il réintroduise le jeu, le dynamisme, que l'édition bi-dimensionnelle amène à gommer. Le système nie l'identité plurielle des folios, leurs déplacements incessants et leurs réutilisations successives. Ce mode d'édition, dépourvu de l'établissement conjoint d'un véritable parcours génétique, revient donc à immobiliser l'écriture dans une chronologie uni-dimensionnelle qui perd son sens. À cela s'ajoute le parti pris méthodologique rigide du «Corpus » qui n'envisage jamais la possibilité d'une composition syntagmatique d'ampleur, une fois le stade des scénarios d'ensemble franchi. On aura l'occasion d'y revenir.

Le second type d'édition verticale intégrale se heurte à un obstacle d'un autre genre : «l'édition téléologique reste peu utilisable pour les recherches macrogénétiques qui portent sur l'évolution des structures de l'avant-texte. » Or, rendre compte des évolutions structurelles paraît être un point fondamental lorsqu' on entreprend l'étude d'un processus d'écriture. Pourtant, la thèse de Pierre-Marc de Biasi contient un tableau très intéressant, dit «Tableau dynamique», qui semblerait justement apte à pallier les insuffisances du «Corpus ». Néanmoins, son examen révèle que seule la dimension paradigmatique (téléologique, comme le dit l'auteur) y est lisible.

Il apparaît donc que le point de rencontre des deux types d'éditions verticales décrits n'est pas tant l'intégralité du corpus qu'elles étudient que leur seule prise en compte de la dimension paradigmatique du travail d'écriture. De ce point de vue, au niveau de la méthode, ces éditions ne se différencient donc pas essentiellement de travaux menés sur des corpus de moindre ampleur, comme ceux de Raymonde Debray Genette, Peter Michael Wetherill, et bien d'autres à leur suite. En effet, bien que ces derniers ne se donnent pas pour but premier l'édition des fragments qu'ils analysent, ils ont souvent joué ce rôle lorsqu'ils travaillaient sur des manuscrits inédits. D'autre part, ils disent plus ou moins explicitement qu'ils opèrent un découpage au sein du processus d'écriture afin de focaliser leur attention sur les étapes qui conduisent à un morceau particulier du texte définitif. Pierre-Marc de Biasi, dans sa thèse, se donne quant à lui pour but de retracer la genèse de chacun des segments du texte définitif de La Légende de saint Julien l'Hospitalier; ce qui revient à multiplier les découpages du type précédemment décrit 26 . Enfin G. Bonaccorso, en partant du principe que les numérotations autographes identiques se suivent génétiquement, privilégie des enchaînements paradigmatiques discutables 27 . Tous ces travaux, en dépit de leur diversité, ont donc en commun leur orientation paradigmatique primordiale.

\section{Clôture du corpus}

Au point où nous en sommes, qu'en est-il de la seconde catégorie créée par Pierre-Marc de Biasi, à savoir celle de l'édition verticale partielle ? Ce que souligne le critique à propos des travaux de Jeanne Goldin 28 , c'est d'abord la

\section{Ibid., p. 186.}

23. Pour les références exactes, voir la note 1 .

24. Pour la référence exacte, voir la note 4.

25. P. 186.

26. On ne prend en compte ici que ce qui se dégage de la lecture des tableaux. Car, dans les explications qu'il donne de la genèse du conte, Pierre-Marc de Biasi dépasse souvent la seule dimension paradigmatique. 27. Voir à ce propos l'ouvrage limpide de Georges A. Willenbrink, «Un coeur simple » : Remarques sur l'avant-texte, Amsterdam-Atlanta, Rodopi, 1989. 
possible incomplétude du corpus considéré; puis, il met en doute son autonomie :

Comment démontrer qu'aucun élément ne manque ? Est-il vraisemblable que l'évolution de ce segment narratif suive une logique autonome indépendante du reste de l'avant-texte? L'édition verticale partielle ne semble pouvoir se pratiquer en toute sécurité que dans le cadre d'un dossier entièrement analysé 29

Les deux questions posées ne sont pas du même ordre. La première, celle de l'incomplétude éventuelle du corpus, se résout à l'aune de l'honnêteté du travail fourni et du temps passé à l'effectuer. Feuilleter, avec une attention extrême, 3500 pages prend du temps, mais le corpus ainsi constitué n'a pas moins de valeur et ne se prête pas plus au reproche d'incomplétude que n'importe quel autre recueil, composé par des mains diverses et dont les capacités scientifiques ne sont pas toujours avérées $30 \ldots$

La deuxième question a bien sûr un enjeu tout autre, c'est celui de l'autonomie des différents mouvements de rédaction. Le problème se pose en des termes différents suivant le niveau où l'on se situe. Il suffit de regarder les scénarios généraux d'un roman pour se rendre compte que l'épisode que l'on a choisi d'étudier fait partie d'un tout, et qu'en tant qu'élément constitutif, il ne bénéficie à ce stade d'aucune autonomie. Pièce parmi les autres pièces, il subit les aléas du jeu de construction. En revanche, la connaissance de la correspondance de Flaubert, et particulièrement des lettres qui forment un «journal de la rédaction» des diverses œuvres, permet de souligner, au niveau de la totalité d'un roman, l'existence de plusieurs périodes d'écriture relativement autonomes. Le passage d'un chapitre à un autre, par exemple, constitue pour Flaubert une véritable rupture dans le processus rédactionnel. Aussi est-ce dans ces moments qu'il préfère effectuer un voyage en Angleterre, ou un séjour à Paris... Il semble donc qu'une fois le stade des scénarios d'ensemble dépassé, on puisse isoler des mouvements au sein du travail rédactionnel, sans pour autant contrevenir à l'idéal, c'est-à-dire à l'unité de conception du roman. Car Flaubert s'il veut «être comme Dieu » n'en a pour autant ni l'omnipotence ni l'étendue du regard... Il doit fractionner pour composer. Chaque chapitre au sein d'un roman ${ }^{31}$ définit donc, selon nous, un mouvement rédactionnel autonome et significatif au sein duquel peut être étudié le processus d'écriture de Flaubert. Le chapitre est conçu, au stade de sa rédaction, comme un tout, un ensemble clos.

\section{Pour une génétique bi-dimensionnelle de l'ensemble clos}

Une fois l'existence de ces mouvements posée, il semble que l'on puisse revisiter les distinctions opérées par PierreMarc de Biasi. En effet, si l'édition verticale partielle pose problème et se différencie de l'édition verticale intégrale, ce n'est que dans l'hypothèse où elle ne parviendrait pas à fonder avec suffisamment de certitude la clôture du mouvement sur lequel porte son étude, c'est-à-dire lorsqu' on peut à bon droit douter de sa capacité à rendre intégralement compte d'un processus d'écriture. En revanche, si cette condition est remplie, ce type d'édition est intrinsèquement apte à permettre une analyse du processus d'écriture. Encore faut-il ne pas tomber dans l'ornière du seul rendu paradigmatique, ce qui n'est pas le cas de Jeanne Goldin 32 .

En effet, le critère de la complétude du corpus (une œuvre entière ou l'une de ses subdivisions) ne paraît pas essentiel pour discriminer théoriquement les types d'éditions génétiques, du moins lorsqu'on s'intéresse essentiellement à la strate des brouillons. À ce critère quantitatif, on préférerait substituer un critère qualitatif, à savoir le respect ou non de la double dimension de l'écriture flaubertienne, de ses orientations concurremment paradigmatique et syntagmatique. Ce critère permettrait de distinguer deux types d'éditions verticales 33 : d'une part, des éditions d'ensembles clos auxquels on aurait restitué le dynamisme de

28. Jeanne Goldin, Les Comices agricoles de Gustave Flaubert, transcription intégrale et genèse dans le manuscrit g223, Droz, 1984; 2 vol. 29. Pierre-Marc de Biasi, «Édition horizontale... », p. 188.

30. On ne sait pas dans quel état étaient les dossiers de Flaubert au moment de sa mort, et quelle a été, par exemple, la part de sa nièce Caroline dans leur constitution. Songeons aussi au premier plan connu d'Un cœur simple qui se trouve «rangé» dans les dossiers de Bouvard et Pécuchet...

31. Le problème est cependant plus aigu pour Madame Bovary qui a connu un découpage en chapitres très tardif...

32. «Nos tableaux d'ordonnance montrent, dans la rédaction des Comices, de longs enchaînements. Le texte se continue, de page à page, avec enjambement syntaxique, phrastique ou diégétique et pagination continue. [...] Flaubert fait alterner ces larges mouvements rédactionnels à pagination continue, avec le paragraphisme si souvent constaté », op. cit., tome 1, p. $46-47$. 
leur écriture par l'établissement d'un parcours génétique bi-axial complet et, d'autre part, des éditions segmentales (ou fragmentales) dont l'orientation est uniquement paradigmatique. Dans le premier type, on trouve l'étude de Jeanne Goldin, mais aussi, phénomène plus curieux, la conjonction a posteriori du «Corpus Flaubertianum I» et du livret que Georges Willenbrink ${ }^{34}$ lui consacre, l'un proposant l'édition des brouillons et l'autre le récit de genèse. Les travaux de ce type devraient se multiplier 35 . Le deuxième genre regroupe quant à lui toutes les autres éditions verticales, ainsi que les analyses ponctuelles de manuscrits précédemment décrites, dont l'utilité et la productivité ne sont plus à démontrer.

\section{La notion de phase angulaire}

Mais, lorsqu' on décide de conduire une étude de genèse sur un ensemble clos, une fois constitué le corpus correspondant à la totalité des brouillons concernés, disons un chapitre, comment repérer et décrire le processus d'écriture, comment parvenir à classer génétiquement toutes ces pages en respectant le rythme propre à la conception de Flaubert? Tentons d'apporter quelques éléments de réponse.

\section{Une structure ternaire récurrente}

Si l'on peut définir le chapitre flaubertien comme un ensemble clos, il va de soi qu'il n'a cependant pas une texture homogène 36 . Lui aussi se décompose en un certain nombre de périodes dont la structure se répète. Ainsi, on a pu mettre en valeur la succession récurrente de trois moments au sein de chaque phase d'élaboration cohérente et isolable.

Le premier moment est une phase prospective d'élaboration scénarique. L'ampleur de cette phase est variable, mais dépasse toujours celle de la séquence concernée par l'élaboration textuelle du deuxième moment. Elle peut ainsi atteindre la totalité du chapitre, ce qui est bien sûr le cas pour les premiers scénarios d'ensemble. Mais le phénomène ne se reproduit pas toujours (une fois sur deux en moyenne). Dans la figure 3 , cette conception scénarique se développe sur un axe vertical qui symbolise la dimension syntagmatique du processus d'écriture, celle de la conti- guïté narrative. Dans la mesure où elle est orientée vers l'aval du texte, on la qualifie de «prospective».

Le deuxième moment est celui de l'élaboration rédactionnelle paradigmatique. Il concerne le processus de textualisation d'un nombre variable de fragments situés au tout début de l'étape de conception scénarique qui précède. Dans la figure 3, l'axe horizontal matérialise ce développement paradigmatique relatif. Relatif en effet, car il est rare que l'on puisse dissocier complètement l'élaboration sur l'axe de la similarité, de la dimension syntagmatique caractéristique d'un enchaînement narratif sur plusieurs folios. On y reviendra.

Le troisième et dernier moment de chaque phase est celui du «recopiage», terme employé par Flaubert luimême. Il concerne au premier chef le texte des folios élaborés dans la séquence précédente. Dans la figure 3 , il se développe sur l'axe vertical de la contiguïté narrative. Néanmoins, le recopiage présente généralement la particularité de ne pas commencer exactement à l'endroit où l'écrivain avait mis en chantier l'élaboration rédactionnelle précédente. Flaubert reprend presque toujours en amont de ce point, recopiant ainsi une ou deux pages de la mise au net antérieure. Bien qu'une commune orientation syntagmatique rapproche ce dernier moment du premier (celui de l'élaboration scénarique prospective), le fait qu'il commence par retourner sur ses pas avant d'enchaîner avec la portion textuelle nouvellement rédigée, le fait qualifier de «rétrospectif».

\section{La modélisation du processus ternaire : la notion de "phase angulaire»}

Quand on reporte l'enchaînement de ces trois moments sur une représentation graphique, on dessine dans l'espace

33. Nous conservons bien sûr la terminologie générale mise en place par Pierre-Marc de Biasi.

34. Georges A. Willenbrink, op. cit.

35. Plaidoyer pro domo puisque je prépare actuellement une thèse de doctorat consacrée à l'étude génétique et critique du chapitre V de Bouvard et Pécuchet.

36. Voir la lettre de Flaubert à Mme Roger des Genettes du 1er mars 1878 : «Tout cela pour le passage que je vais faire, lequel dépend d'une division de mon chapitre, qui pourrait s'intituler : "De la critique historique", laquelle division n'aura pas plus de dix pages », Édition du Club de l'Honnête Homme, tome 5, 1975, p. 36. 


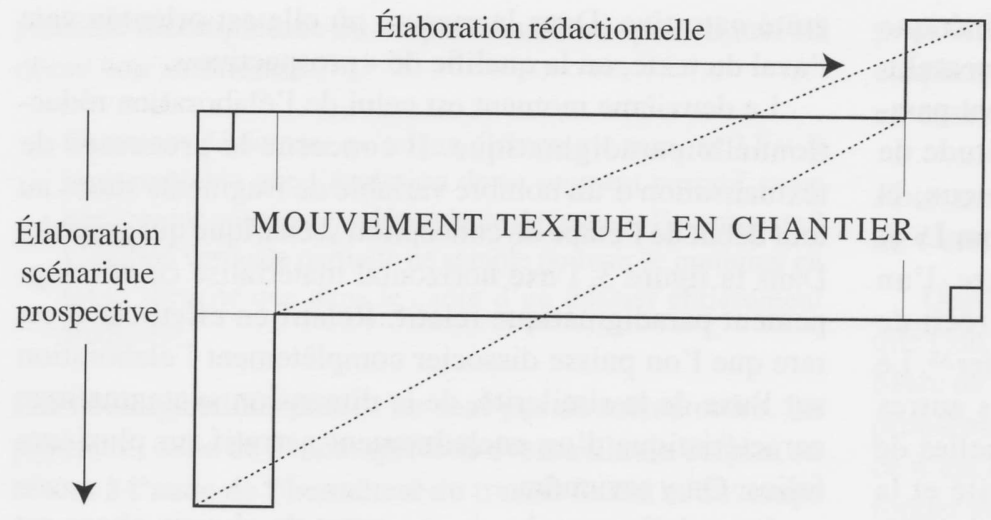

Fig. 3 :

Modélisation

d'une phase angulaire deux triangles rectangles dont la position de l'angle droit varie. Le premier triangle, dont l'angle droit se situe en haut et à gauche, articule la phase d'élaboration syntagmatique des scénarios avec l'étape rédactionnelle paradigmatique. Le deuxième triangle rectangle (dont l'angle droit se situe en bas et à droite) est quant à lui constitué par la rencontre de la phase d'élaboration rédactionnelle avec le recopiage. Le choix terminologique de «phase angulaire» s'explique donc par la récurrence de cette forme géométrique simple.

L'intérêt de cette représentation dans l'espace est de matérialiser la double orientation constante du processus d'écriture flaubertien. D'un côté, l'écrivain est toujours soucieux d'accroître le niveau d'élaboration scénarique de ce qui reste à écrire. De l'autre côté, dès qu'une séquence textuelle est apte à connaître l'étape du recopiage, Flaubert éprouve l'unité et le suivi du texte déjà rédigé, en recopiant cette séquence d'un seul mouvement avec ce qui l'a directement précédée. Il tente donc de faire tenir ensemble, de cimenter avec le plus de cohérence possible, la séquence textuelle qu'il est en train de travailler, ce qui la suit et ce qui l'a précédée 37 .

\section{Comment déterminer l'ampleur d'un enchaînement syntagmatique?}

Mais dans la pratique, face à un dossier en cours de classement duquel on a extrait tous les folios scénariques, comment faire pour déterminer l'étendue variable des diffé- rents enchaînements syntagmatiques qui se succèdent, et comment les articuler avec les divers moments d'élaboration textualisante? On distingue deux phases. Dans la première, on ne s'intéresse qu'au phénomène de contiguïté. On constitue des suites de feuillets dont les contenus narratifs autorisent la succession. Mais il apparaît, au terme de cette opération, que plusieurs feuillets se trouvent souvent en concurrence pour une même place. On introduit alors un critère supplémentaire qui est la numérotation autographe de l'écrivain. Les enchaînements scénariques se démultiplient alors, en une sorte d'éventail; ils ne sont plus fondés sur la seule contiguïté narrative de leur contenu, mais aussi sur la cohérence de leur numérotation. Une même chaîne doit ainsi être entièrement numérotée en chiffres romains ou par des lettres capitales. Mais il ne faut pas négliger les corrections qui affectent très souvent les pages de scénario : les numérotations peuvent connaître jusqu'à quatre ou cinq rectifications successives. Chacun de ces changements est la trace laissée par l'introduction de la page dans une nouvelle série.

Cette première phase permet la constitution de chaînes relativement longues qui donnent l'impression que leur écri-

37. Voir la lettre de Flaubert à Louise Colet du 22 juillet 1852 : « Je voudrais d'un seul coup d'œil lire ces cent cinquante-huit pages et les saisir avec tous leurs détails dans une seule pensée » (Gallimard, «Bibliothèque de la Pléiade», 1980, tome II, p. 135). 
ture a uniformément précédé toute élaboration rédactionnelle. Pour parvenir à isoler les différentes phases scénariques et les articuler avec les moments de rédaction, il faut construire toute une chronologie interne qui se fonde sur la connaissance de principes invariants chez Flaubert. Tout dépend en fait de sa gestion raisonnée du papier : la quasitotalité des brouillons (au sens usuel du terme, c'est-à-dire par opposition aux volumes de scénarios et plans) sont écrits sur les deux faces. Au XIX ${ }^{\mathrm{e}}$ siècle, le papier est encore un matériau que l'on économise. Or l'un des deux côtés de chaque feuille comporte toujours une croix de saint André (un grand « $\mathrm{X} »$ qui surcharge toute la page). Lors de la constitution des recueils et de leur foliotage, il semble qu' on ait choisi, à la Bibliothèque municipale de Rouen comme à la Bibliothèque nationale, de considérer comme recto le côté qui était vierge de ce signe, assimilé à un processus de correction. Or les études de différents corpus flaubertiens ont mis au jour le sens de cette croix : l'écrivain la trace pour indiquer que le contenu de la page a été recopié ailleurs, qu'elle a donc perdu tout intérêt pour le processus en cours, et que son verso, encore vierge, peut rejoindre la pile des feuilles disponibles.

L'économie de Flaubert et sa gestion régulière de la matière première autorisent donc toutes les déductions de chronologie interne qui suivent. Premier principe : le recto et le verso d'une même feuille n'entretiennent jamais un rapport de contiguïté immédiate. Deuxième principe : le verso 38 d'un feuillet est toujours antérieur à son recto. Troisième principe (extension du précédent) : le folio qui contient la première réécriture du verso d'une page est toujours antérieur au recto de cette page ${ }^{39}$. Le respect simultané de tous ces principes met au jour un réseau complexe de préséances qui, une fois coordonnées, permettent de déceler les moments de rupture entre les différentes phases d'élaboration rédactionnelle.

Prenons le cas d'un feuillet scénarique qui concerne la fin du chapitre et qui ne porte pas la croix de saint André. Il a donc été classé comme recto. D'après les principes énoncés ci-dessus, son verso a été écrit avant lui. Si ce dernier consiste en une étape rédactionnelle relativement avancée du milieu du chapitre, on peut en déduire que la textualisation complète de la première moitié du chapitre a précédé l'écriture de ce feuillet scénarique. Inversement, si ce même scénario avait été un verso et si son recto avait été un brouillon du début du chapitre, on pouvait être certain que le scénario appartenait à l'élaboration scénarique d'ensemble du chapitre, élaboration qui a précédé toute textualisation.

\section{L'orientation paradigmatique relative des séquences rédactionnelles}

Mais on peut aller plus loin dans la modélisation et revenir sur les séquences proprement rédactionnelles dont on a qualifié précédemment l'orientation de «relativement» paradigmatique.

\section{Du «paragraphisme» de Flaubert...}

Le travail paradigmatique suivi d'un segment particulier se rencontre peu souvent. En général, ce sont plusieurs segments, répartis sur plusieurs pages, qui connaissent une transformation paradigmatique simultanée, mais décalée. En effet, lors de cette deuxième séquence de chaque phase angulaire, il s'agit bien de faire passer chaque élément du stade scénarique avancé vers son état textuel définitif : c'est le moment où Flaubert dit qu'il «est aux phrases». Mais cette transformation n'affecte pas successivement les différents éléments issus de l'étape scénarique, les métamorphosant les uns après les autres 40 . Le processus d'élaboration textuelle intéresse le plus souvent une pluralité de fragments 41 , comme on peut le voir en examinant la figure 4.

On retrouve, dans ce schéma, les trois moments de la phase angulaire. Le premier, celui de l'élaboration scénarique, est représenté par les seuls feuillets $\mathrm{X}$ et $\mathrm{Y}$, tandis que les deux moments suivants offrent une évolution bien plus complexe, mettant en œuvre les notions d'hétérogénéité et de prolifération. Précisons d'emblée que la figure 4 présente l'image d'une élaboration rédactionnelle paradigmatique «standard»; car le nombre d'étapes et l'ampleur

38. Le verso est ici matériellement défini par le côté qui porte la croix de saint André.

39. Avant qu'une page ne soit barrée et retournée en vue de l'utilisation de son côté vierge, il faut que sa matière ait été préalablement recopiée ailleurs. 
des développements et des réductions sont bien sûr éminemment variables dans la pratique. Si la matière résiste, si la phrase ne vient pas, le nombre de réécritures augmente. Au contraire, si tout se passe bien, l'écrivain arrive assez vite à un niveau d'élaboration qui le satisfait. Le schéma propose donc un profil moyen dont on va décrire les étapes 42 .

Parvenu au stade de l'esquisse (soit ici deux pages, $\mathrm{X}$ et Y), Flaubert isole la première page et ne s'intéresse qu'à elle. Il en répartit la matière sur trois feuillets, respectivement X1, X2 et X3. Dûment corrigé et amplifié, le contenu d'une page et demie (souvent, Flaubert se fonde pour ses coupures sur une articulation logique du récit) est reporté sur de nouveaux feuillets: $\mathrm{X} 12$ et $\mathrm{X} 2^{2}$. C'est alors que l'écrivain concentre son attention uniquement sur le point de départ de son mouvement, ne reprenant que le début de la première page sur un nouveau feuillet, $\mathrm{X} 1^{3}$ (notons que la matière travaillée dans $\mathrm{X} 1^{3}$ est d'une ampleur inférieure à celle traitée dans $\mathrm{X} 1^{2}$ ). Satisfait du niveau d'élaboration auquel il est parvenu, il recopie une dernière fois le début de son texte sur la page X14. Mais celle-ci ne contient pas seulement la matière mise au point dans le folio $\mathrm{X} 1^{3}$ : elle enchaîne avec les segments abandonnés depuis le feuillet $\mathrm{X} 12$. Ayant rempli matériellement la page X14, Flaubert prend une page $\mathrm{X} 2^{3}$ et une page $\mathrm{X} 3^{2}$ pour retravailler le contenu du folio X22. À ce moment, les folios X2 et X3 (qui demeuraient jusque-là en attente) voient leur numérotation autographe harmonisée avec celle de la séquence $\mathrm{X} 1^{4}-\mathrm{X} 2^{3}-\mathrm{X} 3^{2}$ : ils deviennent, après rature de leur première numérotation, $\mathrm{X} 4$ et $\mathrm{X} 5$.

Flaubert reprend alors son processus rédactionnel : il recopie sur le folio $\mathrm{X} 24$ les segments textuels de la fin de la page $\mathrm{X} 14$ dont il n'est pas satisfait, et seulement ceuxci, auxquels il joint une partie de la matière du feuillet X23 . Aussitôt, il reprend la dernière page écrite sur une nouvelle feuille, X25. C'est alors que la matière des folios X4 et X5 entre à son tour dans la danse. Recopiée, fractionnée et amplifiée à la suite de celle du feuillet X32, elle est distribuée sur quatre nouvelles pages : X333, X42, X52 et X6.

Le processus se reproduit alors comme précédemment. L'écrivain reprend la rédaction au niveau de X $3^{3}$ qu'il recopie une première fois sur un feuillet vierge, $\mathrm{X} 3^{4}$, puis une dernière fois sur le folio $\mathrm{X} 3^{5}$. La matière des pages $\mathrm{X} 4^{2}$, $\mathrm{X} 5^{2}$ et X6 est développée et donne naissance aux folios $\mathrm{X} 4^{3}, \mathrm{X} 5^{3}$ et $\mathrm{X} 6^{2}$. La page $\mathrm{X} 4^{3}$ est travaillée deuX fois successivement, feuillets X44 et X45, ce dernier s'enchâ̂nant avec la page $\mathrm{X} 5^{4}$, reprise des feuillets $\mathrm{X} 5^{3}$ et $\mathrm{X} 6^{2}$.

À ce stade, Flaubert a entièrement rédigé la matière scénarique qui était contenue dans la page d'esquisse X. Le processus de textualisation est achevé, mais Flaubert n'est pas encore satisfait : il faut que cette nouvelle séquence, artificiellement isolée du reste du chapitre pour les besoins de la rédaction, refasse corps avec lui. C'est alors qu'a lieu la troisième séquence de la phase angulaire décrite précédemment, c'est-à-dire le recopiage de ce qui vient d'être rédigé, un recopiage dont l'orientation est syntagmatique, mais qui offre une dimension rétrospective. En effet, l'écrivain recopie son mouvement mais ne commence pas là où avait débuté le processus rédactionnel. Il reprend en amont, recopiant d'abord la matière d'une ou deux pages de la mise au net du mouvement rédactionnel précédent (ici W45),

40. Peter Michael Wetherill offre l'exposé le plus clair de cette conception du travail de Flaubert dans l'introduction de son édition de L'Éducation sentimentale (classiques Garnier, 1984 ; p. LI) : «Les brouillons, par contre, ont pour unité de base des mouvements beaucoup plus brefs, qui tiennent presque toujours dans une seule feuille de papier [...]./ Flaubert part donc d'une fragmentation de son texte. Le tronçon isolé sur lequel Flaubert travaille passe par de nombreuses versions avant de parvenir à son état final. Sur le premier jet, Flaubert opère les corrections qui lui paraissent nécessaires [...]. Il recopie sur une deuxième feuille cette version corrigée et recommence. Cette deuxième ébauche une fois corrigée est de nouveau recopiée et de nouveau recorrigée. Et ainsi de suite, jusqu'à sept, huit, neuf versions. Puis, satisfait, Flaubert passe au «tronçon » suivant. Tout le roman s'écrit ainsi. De temps à autre, Flaubert s'arrête pour recopier le chapitre qu'il vient de terminer. »

41. Dans son ouvrage cité, Jeanne Goldin avait déjà marqué sa défiance vis-à-vis de ce paragraphisme qui était plus celui du critique, que celui de Flaubert... (p. 45-46).

42. Pour souligner la réutilisation des mêmes chiffres par Flaubert dans sa numérotation autographe, et pouvoir néanmoins distinguer les folios, on utilise une numérotation assortie d'un exposant : X14 signifie que le chiffre 1 est utilisé pour la quatrième fois par l'écrivain dans ce mouvement rédactionnel concernant l'esquisse $\mathrm{X}$, et que quatre folios différents portent donc, à ce moment précis, cette même numérotation autographe. Pour revenir à un aspect moins abstrait, il suffit de donner une valeur chiffrée à l'inconnue, et de transformer toutes les formules (si X1 est égal à $10, \mathrm{X} 2$ sera remplacé par 11 et W45 par 9). L'examen de ce tableau prouve qu'on ne peut pas fonder une étude génétique sur le critère de la seule numérotation autographe : non seulement la rédaction des divers folios qui portent le même numéro est séparée par la rédaction d'autres pages, mais surtout le même chiffre recouvre souvent des fragments textuels qui, à cause du glissement de l'écriture, n'ont plus rien à voir. Il n'y a qu'à comparer l'extension des folios $\mathrm{X} 1^{3}$ et X $1^{5}$ pour s'en rendre compte. 


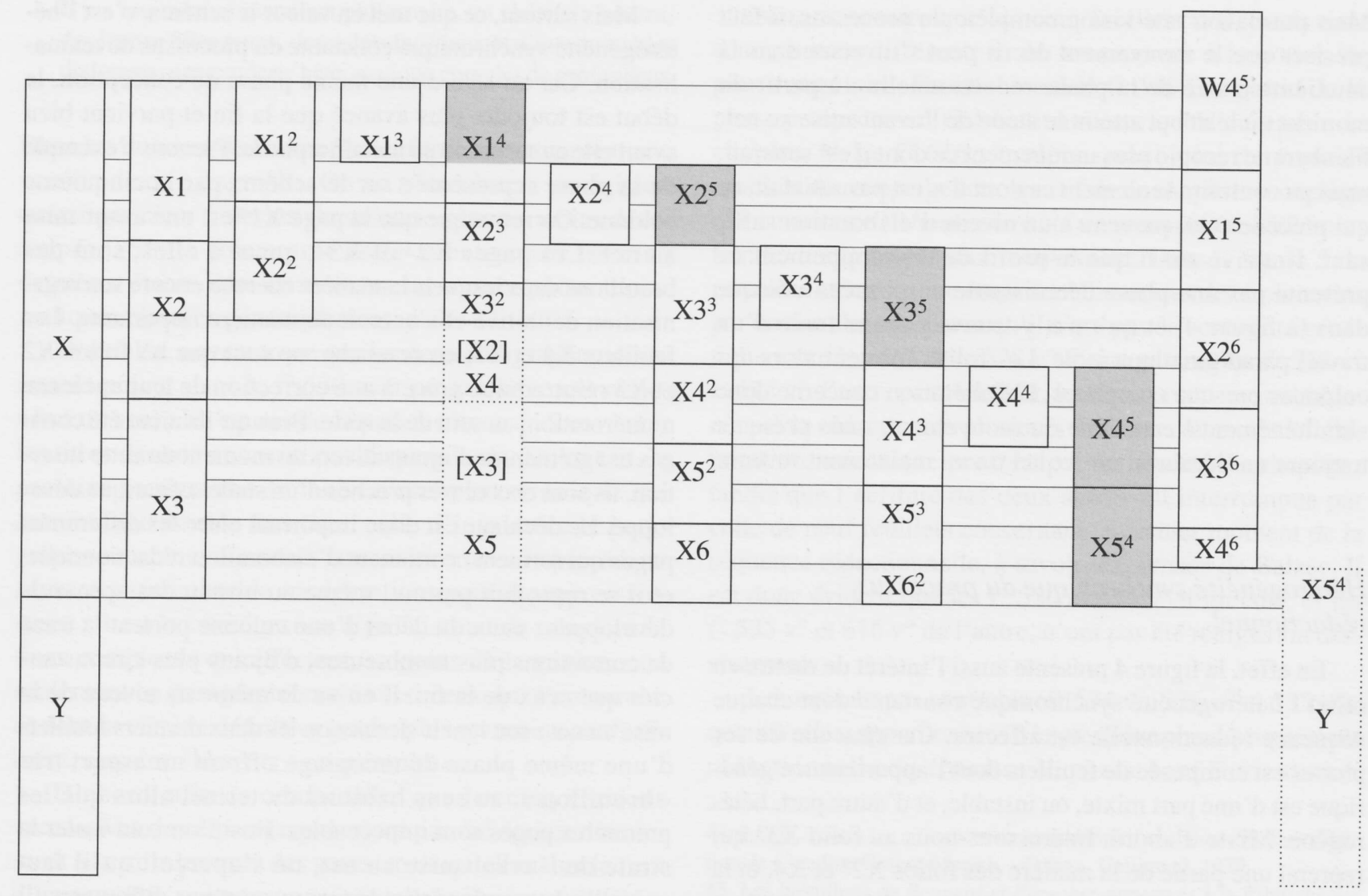

Fig. 4 : Profil de développement d'une séquence rédactionnelle «standard»

auxquelles il enchaîne les quatre feuillets X15, X26, X36 et $\mathrm{X} 46$. Souvent, l'extrême fin du folio X54 n'est pas recopiée : cette transition entre le contenu scénarique des pages $\mathrm{X}$ et $\mathrm{Y}$ est directement reportée en haut de la page $\mathrm{Y}$ et sert «d'embrayeur» à la séquence rédactionnelle qui suit immédiatement, la page Y passant à son tour par toutes les étapes successivement décrites pour la page $\mathrm{X}$.

Ainsi, l'orientation paradigmatique de la phase de textualisation ne peut être dissociée d'une dimension syntagmatique d'ampleur variable, presque constamment associée à chacune de ses étapes. Dans la mesure du possible, Flaubert essaie de travailler concurremment sur de vastes segments textuels. D'autre part, la modélisation présentée ci-dessus souligne combien est juste la notion de «composition en fugue» proposée par Raymonde Debray Genette 43 :

L'autre visée, bien que macrostructurelle, relève encore de l'endogenèse. Elle régit le rapport des pages entre elles, en particulier le point de reprise, car ce n'est pas forcément la fin de la page précédente. Comme Flaubert recopie seulement ce dont il est satisfait, quitte à le retravailler encore, il ne recopie qu'une part de la page précédente et continue tantôt en deçà, tantôt au-delà du point qui l'a terminée. Si l'on tient compte des pages perdues, c'est bien à une composition en fugue qu'on a affaire, mais avec des thèmes incomplets.

43. Métamorphoses du récit, Seuil, 1988; «Esquisse de méthode», p. 30. 
Mais pour avoir une vision complète du processus, il faut préciser que le mouvement décrit peut s'inverser dans la deuxième partie de la phase rédactionnelle : à partir du moment où le début atteint le stade de l'avant-mise au net, Flaubert ne recopie plus uniquement ce dont il est satisfait, mais au contraire seulement ce dont il n'est pas satisfait, ce qui précède étant parvenu à un niveau d'élaboration suffisant. Il arrive aussi que le profil de développement ne présente pas une phase décroissante aussi accentuée que dans la figure 4, et qu'on n'y trouve aucune trace d'un travail paradigmatique isolé. Les folios forment alors des colonnes presque complètes. L'élaboration concerne donc simultanément l'ensemble du mouvement, mais présente toujours un décalage sur lequel on va maintenant revenir.

\section{Hétérogénéité synchronique du processus rédactionnel}

En effet, la figure 4 présente aussi l'intérêt de mettre en relief l'hétérogénéité synchronique constante dont chaque séquence rédactionnelle est affectée. Car chacune de ses phases est composée de feuillets dont l'appartenance générique est d'une part mixte, ou instable, et d'autre part, hétérogène. Mixte d'abord. Intéressons-nous au folio X33 qui reprend une partie de la matière des folios $\mathrm{X} 23$ et $\mathrm{X} 4$, et la totalité du folio X32. Flaubert recopie donc sur une même page des segments textuels dont les premiers ont déjà connu trois élaborations successives alors que les derniers n'en sont qu'à leur première. Il ne serait donc pas étonnant que cette page présente dans sa première partie une textualisation inaboutie encore, certes, mais avancée, tandis que la seconde partie n'offrira qu'une organisation de la matière par syntagmes nominaux.

C'est aussi son instabilité intrinsèque qui rend difficile l'identification générique d'un folio. En effet, l'écriture d'un feuillet comprend au moins deux phases. La première est celle du premier jet : Flaubert recopie ce dont il est satisfait dans la page précédente. Ensuite, au terme du mouvement et avant de le recopier, il relit le folio, le corrige et l'enrichit. Or cette deuxième phase peut faire «basculer» le folio d'une catégorie dans une autre. C'est pourquoi afin d'identifier rigoureusement un folio, il faudrait distinguer entre son premier jet et son état final corrigé.
Mais surtout, ce que met en valeur le schéma, c'est l'hétérogénéité synchronique constante du processus de textualisation. Car au sein d'une même phase de conception, le début est toujours plus avancé que la fin et parvient bien avant elle au terme de sa métamorphose. Prenons l'exemple de la phase représentée sur le schéma par la cinquième colonne. On remarque que la page $\mathrm{X} 14$ est une avant-mise au net. Les pages $\mathrm{X} 2^{3}$ et X $3^{2}$, quant à elles, sont des brouillons dans lesquels la matière cherche encore son organisation définitive et s'accroît de manière importante. Les feuillets X4 et X5, en revanche, ne sont que les folios X2 et $\mathrm{X} 3$ réintroduits, grâce à une correction de leur ancienne numérotation, au sein de la série. Bien qu'ils aient été corrigés et agrémentés d'ajouts divers au moment de cette insertion, ils sont encore très proches d'un stade scénarique développé. Le décalage est donc important entre les différentes pages qui forment ce moment d'élaboration rédactionnelle, et il se reproduit partout, même au niveau des scénarios développés : ceux du début d'une colonne portent la trace de corrections plus nombreuses, d'ajouts plus circonstanciés que ceux de la fin. Il en va de même au niveau de la mise au net : souvent le dernier ou les deux derniers feuillets d'une même phase de recopiage offrent un aspect très «brouillon», au sens habituel du terme, alors que les premières pages sont impeccables. Et si l'on veut isoler la strate de l'avant-mise au net, on s'aperçoit qu'il faut recueillir le premier folio de quatre colonnes différentes (il s'agit des feuillets grisés dans la figure 4). Après ces constats de mixité, d'instabilité et d'hétérogénéité, il apparaît donc impossible de dissocier, en synchronie, des strates homogènes au sein de la couche des brouillons.

\section{Un exemple emprunté au chapitre $V$ de Bouvard et Pécuchet}

À l'appui de ces considérations un peu abstraites, arrêtons-nous sur un cas concret d'élaboration de paragraphe chez Flaubert. Il s'agit d'un extrait du chapitre V de Bouvard et Pécuchet, dont voici la version publiée 44 :

Bouvard n'en continua pas moins Walter Scott, mais finit par s'ennuyer de la répétition des mêmes effets. L'héroïne, ordinairement, vit à la campagne avec son père, et l'amoureux, un enfant volé, est rétabli dans ses droits et triomphe de ses rivaux. 
Il y a toujours un mendiant philosophe, un châtelain bourru, des jeunes filles pures, des valets facétieux et d'interminables dialogues, une pruderie bête, manque complet de profondeur.

Dans les dossiers de Rouen 45 , neuf folios contiennent une version de ce paragraphe. On ne s'intéressera pas ici aux quatre premiers (relevant du scénario plus ou moins développé), afin de concentrer notre attention sur les cinq dernières étapes dont on trouvera les transcriptions diplomatiques classées génétiquement, p. 76-82.

D'emblée, on remarque que les deux paragraphes initialement prévus dans le $\mathrm{f}^{\circ} 530$ sont progressivement ramenés à un seul : les causes de la persévérance de Bouvard et les titres des romans scottiens disparaissent. De ce fait, le premier paragraphe se mue en une phrase unique qui assume une double fonction, celle d'introduire le paragraphe suivant et d'assurer la transition avec le précédent. Mais ce phénomène de condensation apparaît progressivement : il est la conséquence d'un faisceau de transformations complexes qui affectent, au cours de la genèse, non seulement ce paragraphe mais aussi ceux qui l'entourent, et dont la version définitive garde, plus ou moins confusément, certains éléments «en mémoire».

D'abord, afin de comprendre que les cinq versions successives de ce paragraphe n'ont pas été écrites directement à la suite les unes des autres mais qu'elles sont le fruit d'un parcours, quittons une visée trop exclusivement téléologique et replaçons nos cinq feuillets dans le mouvement général de la genèse tel que nous l'avons reconstitué46 dans la figure 5 .

On s'aperçoit tout d'abord que la situation du paragraphe de référence (matérialisé par une zone grisée) fluctue dans l'espace de la page. Elle occupe le dernier quart $\operatorname{des} \mathrm{f}^{\circ} 530$ et $534 \mathrm{v}^{\circ}$, le premier tiers du $\mathrm{f}^{\circ} 536 \mathrm{v}^{\circ}$, le dernier quart du $\mathrm{f}^{\circ} 535 \mathrm{v}^{\circ}$, et le second tiers du $\mathrm{f}^{\circ} 616 \mathrm{v}^{\circ}$. En effet, la portion de texte définitif concernée par les cinq feuillets est très variable suivant les cas : pour le $\mathrm{f}^{\circ} 530$, onze paragraphes en amont du paragraphe de référence 47 ; pour le $f^{\circ} 534 \mathrm{v}^{\circ}$, six paragraphes en amont 48 ; pour le f $536 \mathrm{v}^{\circ}$, trois paragraphes en aval 49 ; pour le $\mathrm{f}^{\circ} 535 \mathrm{v}^{\circ}$, cinq paragraphes en amont 50 ; enfin, pour le $\mathrm{f}^{\circ} 616 \mathrm{v}^{\circ}$, quatre paragraphes en amont et trois en aval51. L'identité et le nombre de paragraphes travaillés simultanément sur une même page sont donc éminemment variables d'un feuillet à l'autre.
Notons ensuite que les cinq feuillets concernés sont d'une nature différente si on les rapporte à la notion de phase angulaire. En effet, seul le f ${ }^{\circ} 530$ fait partie de la phase prospective d'élaboration scénarique d'ensemble du chapitre $\mathrm{V}$. Le $\mathrm{f}^{\circ} 616 \mathrm{v}^{\circ}$ relève quant à lui du premier moment de recopiage rétrospectif de ce chapitre, recopiage qui sanctionne l'aboutissement (momentané) de l'élaboration textuelle du premier mouvement. Dans la figure 5, la première et la dernière étape sont dissociées des trois autres par un double trait, parce qu'il n'y a pas de continuité génétique entre les $\mathrm{f}^{\circ} 532$ et $528 \mathrm{v}^{\circ}$ d'une part, et les $\mathrm{f}^{\circ} 555 \mathrm{v}^{\circ}$ et $673 \mathrm{v}^{\circ}$ d'autre part. En effet, les deux premiers sont séparés par la rédaction de plusieurs feuillets consacrés à l'élaboration scénarique prospective de la suite du chapitre; tandis que l'écriture des deux autres est interrompue par celle de neuf feuillets concernant le dernier moment de la séquence rédactionnelle, à savoir les romans de Balzac. Il est donc évident que les f 530 et 534 v $^{\circ}$ d'un côté, et les $\mathrm{f}^{\circ} 535 \mathrm{v}^{\circ}$ et $616 \mathrm{v}^{\circ}$ de l'autre, n'ont pas été rédigés successivement.

Les trois étapes centrales $\left(\mathrm{f}^{\circ} 534 \mathrm{v}^{\circ}, 536 \mathrm{v}^{\circ}\right.$ et $\left.535 \mathrm{v}^{\circ}\right)$, quant à elles, appartiennent à la première phase proprement rédactionnelle du chapitre $\mathrm{V}$, phase dont l'orientation

44. P. 203. Toutes les références au texte définitif sont données dans l'édition de Claudine Gothot-Mersch, «Folio», Gallimard, 1979.

45. Les brouillons de Bouvard et Pécuchet, conservés à la Bibliothèque municipale de Rouen, ont connu récemment une nouvelle reliure; ils ont été redistribués en neuf volumes selon une foliotation continue. Ceux qui concernent le chapitre $\mathrm{V}$ du roman se trouvent rassemblés, à quelques exceptions près, sous les cotes g225 (5) et g225 (6). Je remercie Mlle Rose, Conservateur général de la Bibliothèque, de m'avoir autorisée à les consulteret à en reproduire certains ici-même.

46. Dans l'espace alloué, il est impossible de justifier pleinement cet enchaînement génétique. Précisons seulement qu'il est fondé, entre autres, sur l'examen de la numérotation autographe (et de ses remaniements), ainsi que sur les résultats obtenus à partir des trois principes «économiques » mis en lumière précédemment (p. 71).

47. P. 201-203; de «Ils lurent d'abord Walter Scott... » jusqu'à "... manque complet de profondeur.»

48. P. 202-203; de «Après Walter Scott, Alexandre Dumas... » jusqu'à «... manque complet de profondeur.»

49. P. 203-204; de «Bouvard n'en continua pas moins... » jusqu'à « ... et des conspirations de Cellamare!»

50. P. 202-203; de «Rendus difficiles par ces deux maîtres... » jusqu'à «... manque complet de profondeur.»

51. P. 202-204; de «La couleur de Frédéric Soulié... » jusqu’à «... une foule de Jeanne d'Arc. » 
Ainsi-dans Guy Manering dan l'antiquire 'L'Héroïne n'a pas de mère a est élevée par son à la campagne.

père. l'amoureux est un enfant, ou volé rétabli dans ses droits $\alpha$ sa fortune, $\alpha$ qui Les personnages secondaires meilleurs mais toujours les mêmes-

en vient toujours aux mains avec son rival. Preque toujours un mendiant philosophe - héros d'opéra-comique toutes les vertus $\alpha$ insignifiant $\ldots . .$. convenables.

invariablemt une vieille Bohémienne effrayante d'abord mais bonne ensuite. des jeunes filles les valets plais facétieux $\alpha$ dévoués pures. un vieux seigneur, bourru bienfaisant, un personnage mystérieux - héreune d'on - longueur intolérable des dialogues. quand trois d/lignes d'action suffiraient,

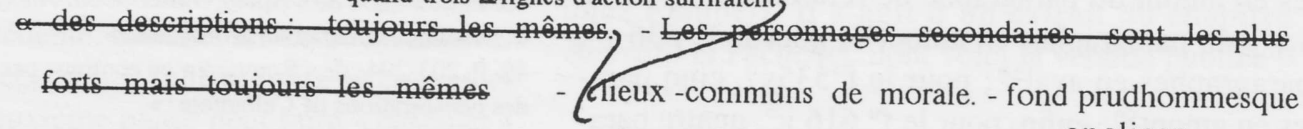




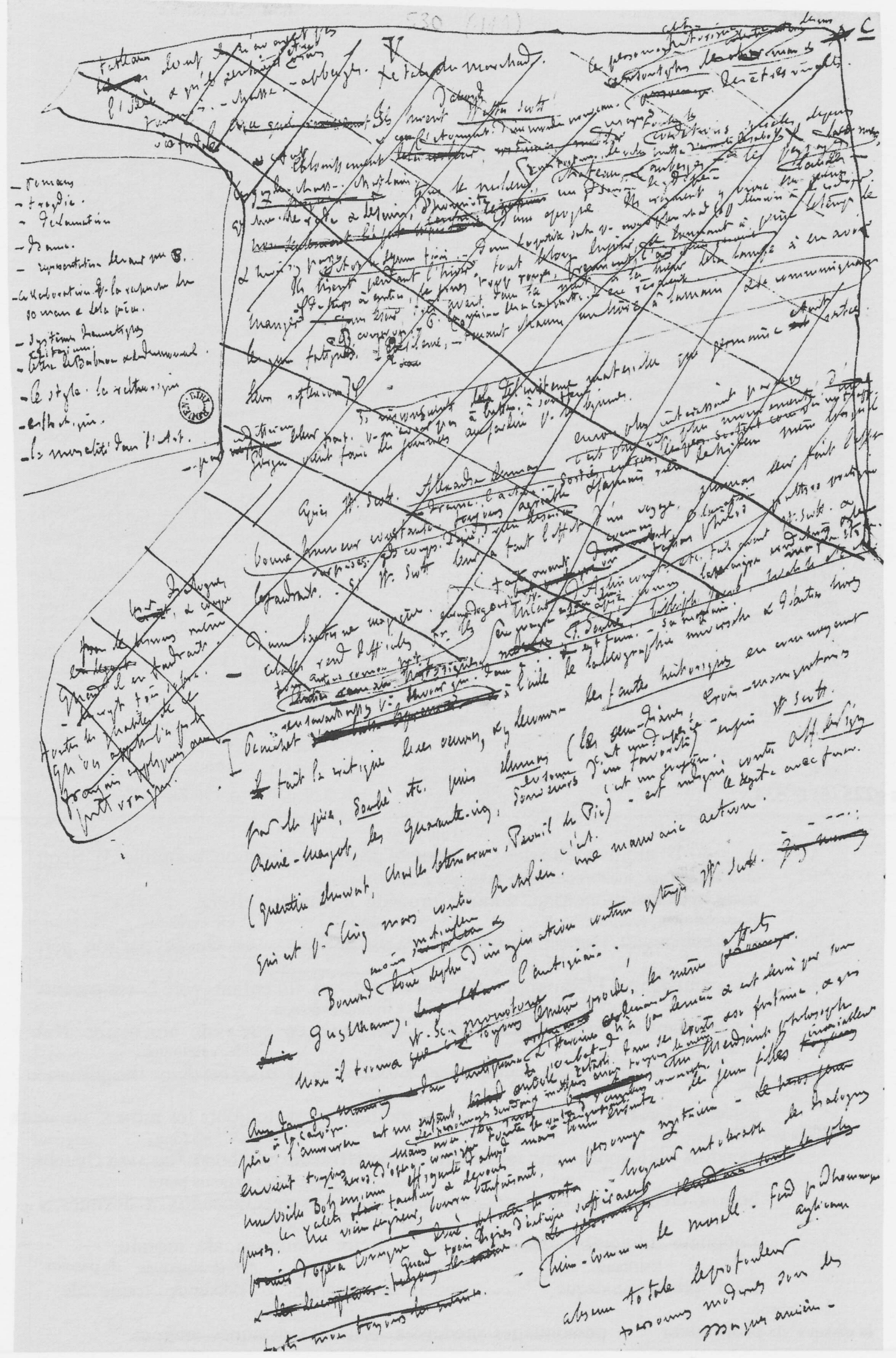

Fig. 6 : Ms g225 (5) fo 530 (Bibliothèque municipale de Rouen) 
- B. moins méticuleux. a plus d'imagination continue W. Scott Guy Man., l'ant., mais s'ennuya de la longueur des dialogues. maisle trowe monotone, comme procédé a comme effets. B. ordinair. erdinair. vit avec à la campagne - L'amoureux rétabli dans a triomphé de son rétabli dans ses droits en être venu aux mains avec

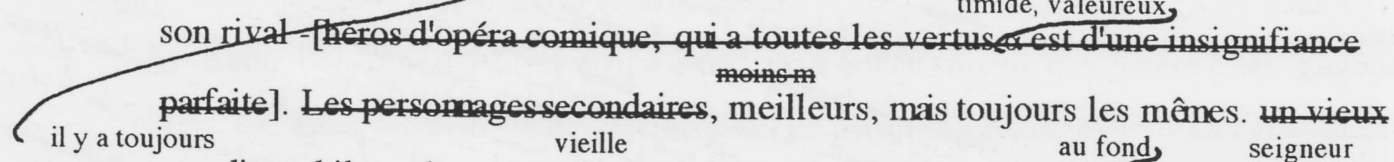

mendiant philosophe, une tielle bohémienne, effrayante mas bonne tun viax châtelain

jeunes filles toujours pures

bourru bienfaisant, un personnage mystérieux, valets facétreux $\alpha$ devourés.

29 Longueur-intérable des dialogues - lieux communs de morale,
30 lourdaud. absolu. 


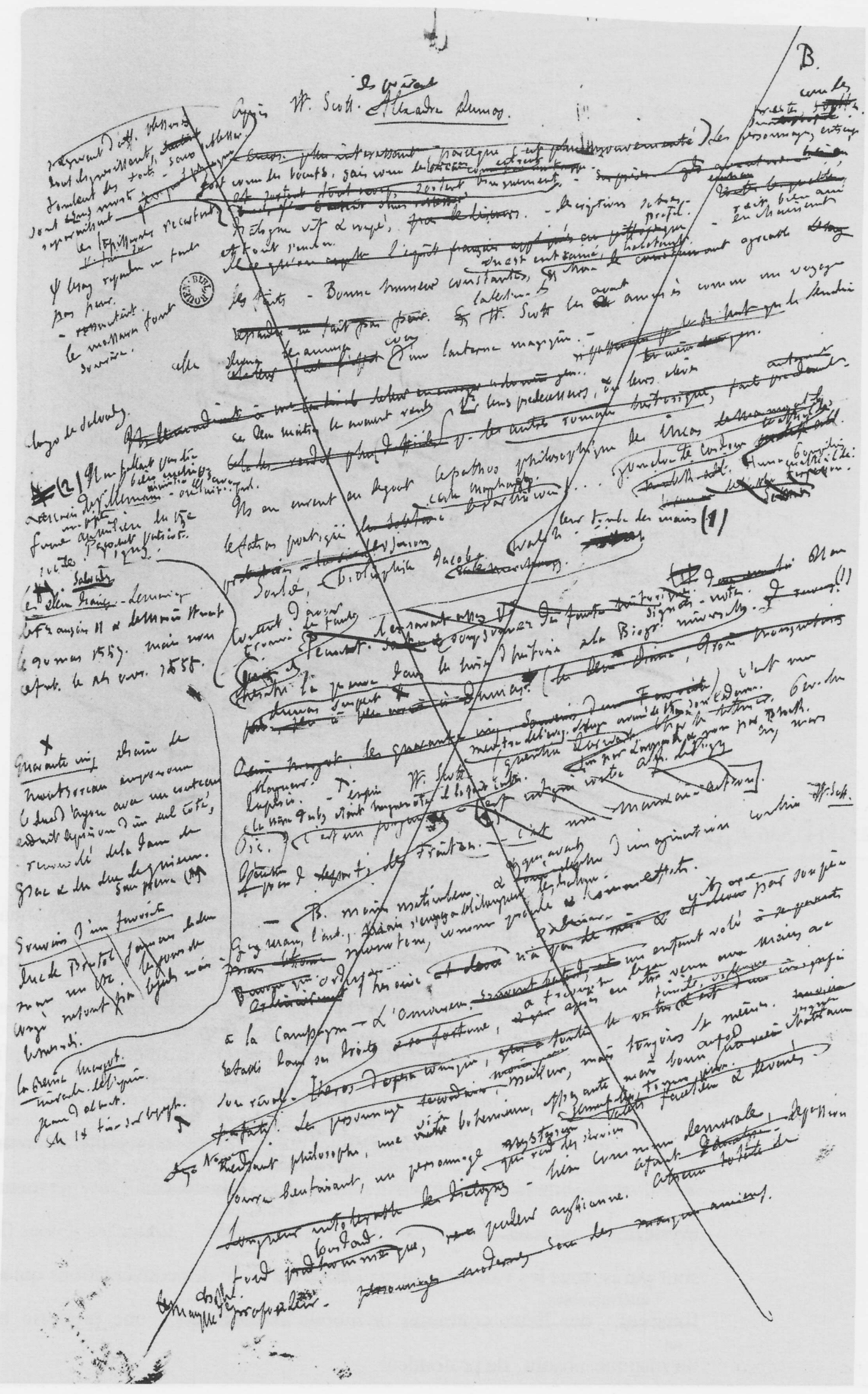

Fig. 7 : Ms g225 (5) $\mathrm{f}^{\circ} 534 \mathrm{v}^{\circ}$ (Bibliothèque municipale de Rouen) 


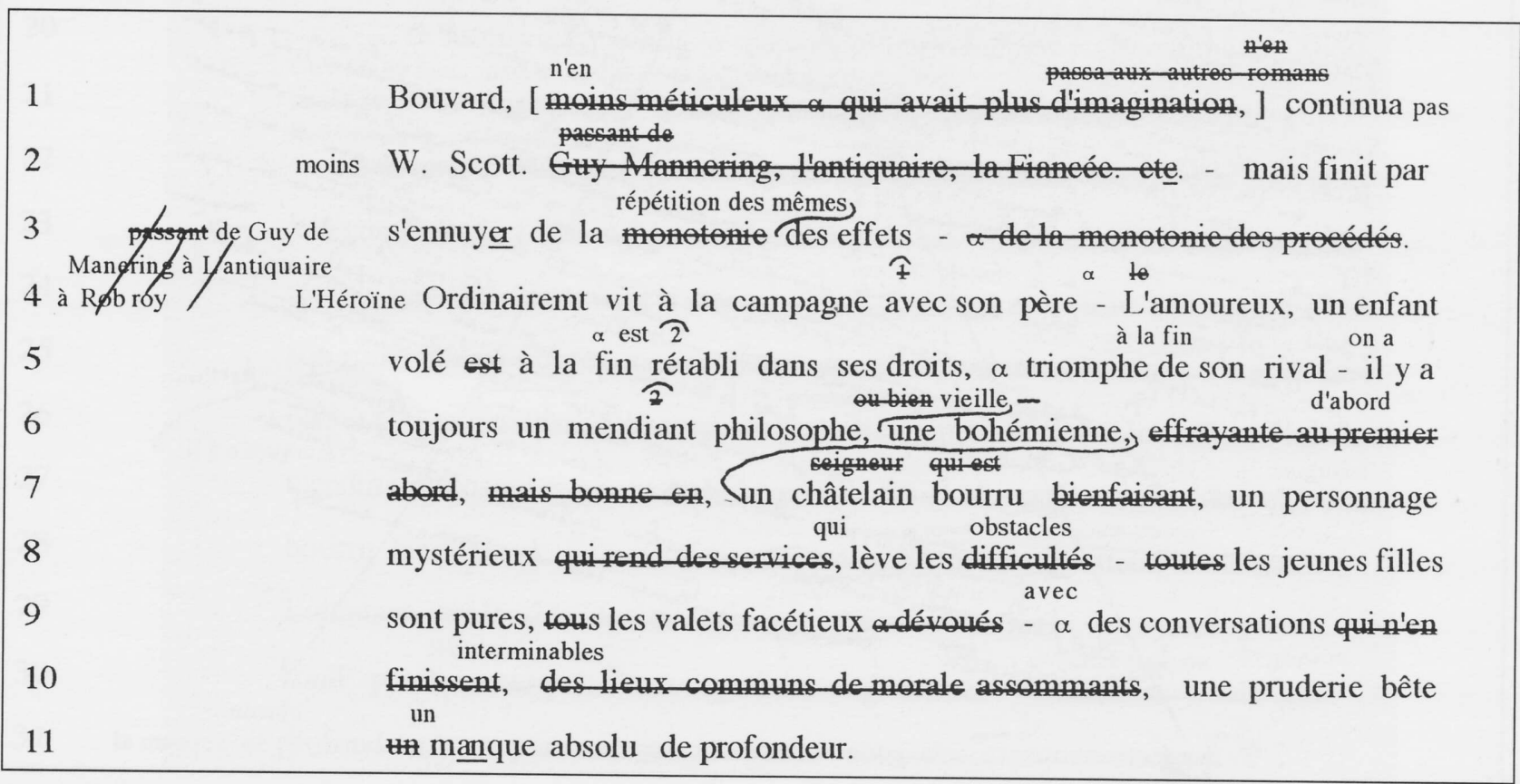




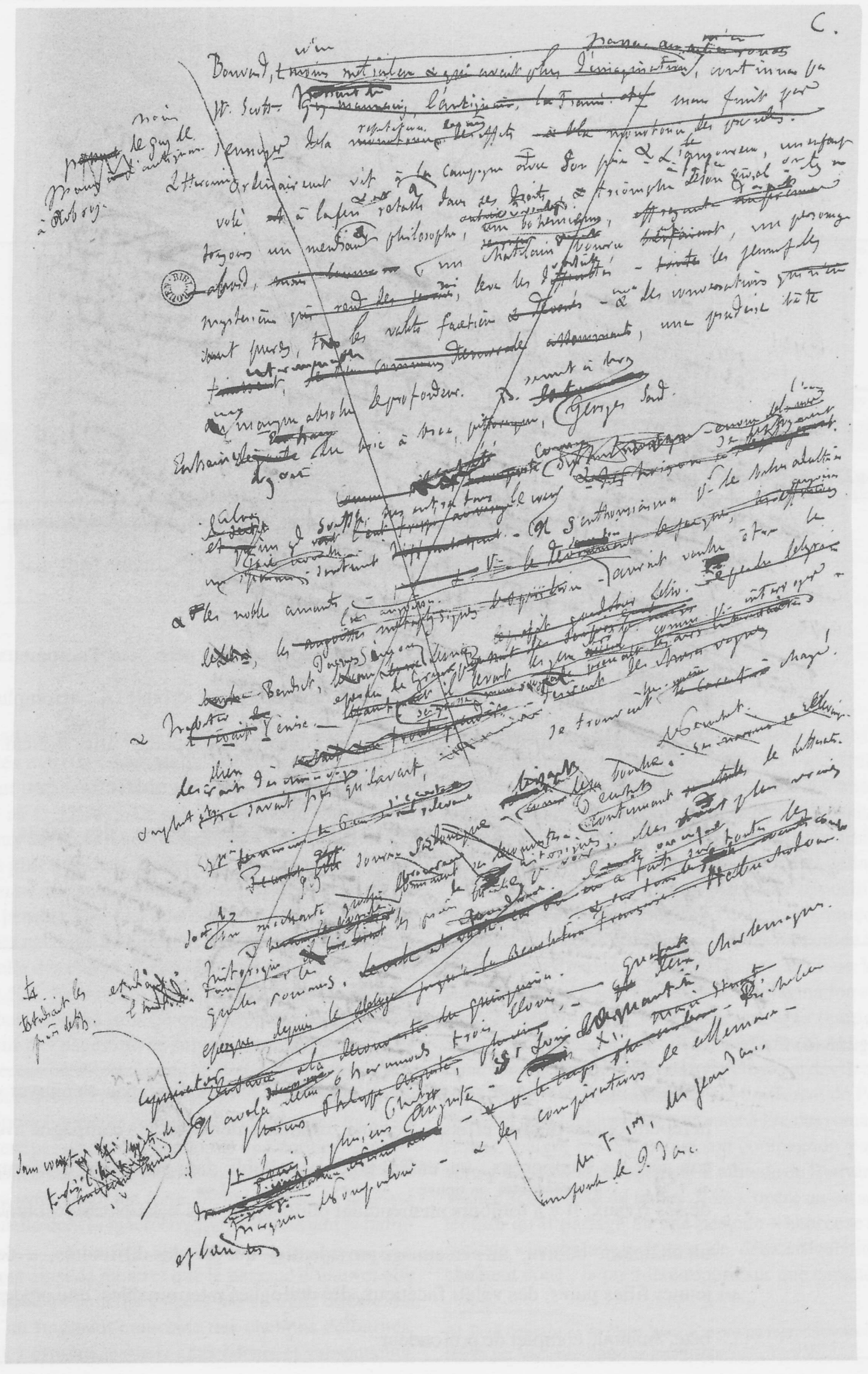

Fig. 8 : Ms g225 (5) $\mathrm{f}^{\circ} 536 \mathrm{v}^{\circ}$ (Bibliothèque municipale de Rouen) 


\begin{tabular}{|c|c|}
\hline 29 & Bouvard n'en continua pas moins W. Scott, \\
\hline 30 & RobRoy. W \\
\hline 31 & s'ennuyer de la répétition des mêmes effets. \\
\hline 32 & $\begin{array}{l}\text { L'Héroüne, ordinairement vit à la Campagne avec son père - } \alpha \text { l'amoureux } \\
\text { enfant }\end{array}$ \\
\hline 33 & 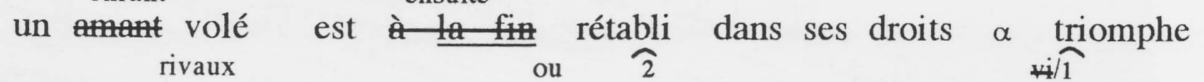 \\
\hline 34 & de son il y a toujours un mendiant philosophe, une bohém. \\
\hline 35 & $\begin{array}{l}\text { secourable, un châtelain bourru, un personnage mystérieux qui } \\
\text { ôte }\end{array}$ \\
\hline 36 & tes difficultés, $\frac{\alpha}{2}$ des jeunes filles pures, des valets \\
\hline 37 & facétieux, des dialogues interminables, une pruderie bête, un \\
\hline 38 & $\underset{\text { complet }}{\operatorname{manque}} \underset{\text { absolu de profondeur. }}{ }$ \\
\hline
\end{tabular}

$5^{\circ}$ Ms $\mathrm{g} 225(6) \mathrm{f}^{\circ} 616 \mathrm{v}^{\circ}$

Bouvard n'en continua pas moins Walter Scott, mais finit par s'ennuyer de la répétition des mêmes effets. L'hérö̈ne, ordinairement, vit à la campagne avec son père, $\alpha$ l'amoureux, un enfant volé est rétabli dans ses droits $\alpha$ triomphe on trouve [थ de ses rivaux. Il y a toujours un mendiant philosophe, une bohémienesecouble aplanit un châtelain bourru, des jeunes filles pures, des valets facétieux, des dialogues interminables, une pruderie bête, manque complet de profondeur. 


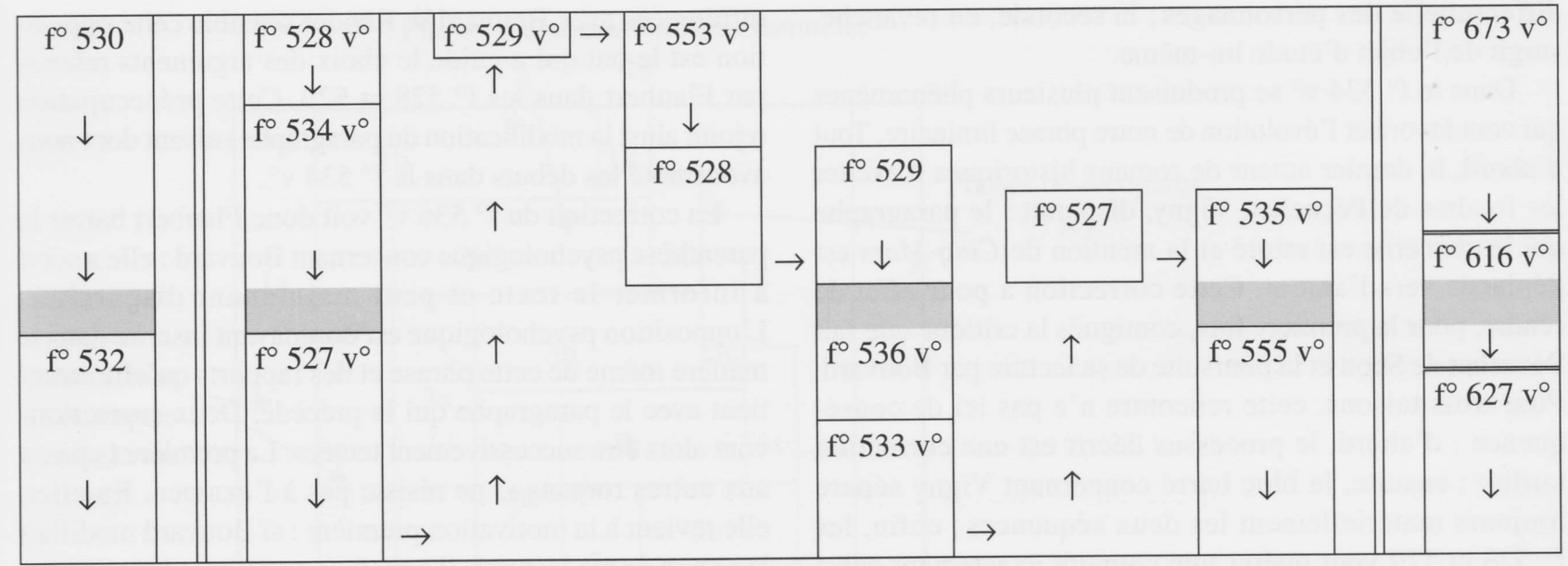

Fig. 5 : Une séquence rédactionnelle du chapitre V de Bouvard et Pécuchet

paradigmatique relative nous intéresse ici. Les douze folios représentés entre les deux doubles barres de la figure 5 constituent un enchaînement génétique ininterrompu (du $\mathrm{f}^{\circ} 528 \mathrm{v}^{\circ}$ au f $\mathrm{f}^{\circ} 555 \mathrm{v}^{\circ}$ ). Or même au sein de cette phase, jamais deux des trois feuillets concernés ne sont écrits à la suite (pas plus que dans les deux cas analysés précédemment, comme une analyse purement téléologique aurait pu le laisser penser). La rédaction des $\mathrm{f}^{\circ} 534 \mathrm{v}^{\circ}$ et $536 \mathrm{v}^{\circ}$ est séparée par celle des feuillets $527 \mathrm{v}^{\circ}, 529 \mathrm{v}^{\circ}, 553 \mathrm{v}^{\circ}, 528$ et 529 ; celle des fo $536 \mathrm{v}^{\circ}$ et $535 \mathrm{v}^{\circ}$ par celle des feuillets $533 \mathrm{v}^{\circ}$ et 527 . Néanmoins, précisons-le, il arrive ailleurs que Flaubert réécrive trois ou quatre fois la même portion de texte sur des pages qui se suivent immédiatement. Ces cas de succession directe, rares au demeurant, n'apparaissent que lorsque l'écrivain rencontre une résistance particulière. Sporadiques, ils n' ont d'autre valeur que locale et ne remettent pas en cause l'économie de l'écriture flaubertienne fondée sur la persistance microstructurelle d'une dimension syntagmatique forte, même au sein de la phase rédactionnelle dont la macrostructure est pourtant paradigmatique.

On vient ainsi de montrer que le passage d'une version de paragraphe à l'autre ne s'opère pas en ligne directe. La genèse d'un fragment emprunte des chemins détournés, traverse des espaces textuels antécédents et subséquents, des espaces qui définissent un véritable «contexte » à chaque évolution particulière. Une fois l'existence de ce parcours posée, venons-en maintenant à l'analyse du parcours spécifique conduisant de la phrase introductive de notre paragraphe de référence telle qu'elle apparaît dans le $f^{\circ} 530$ à celle qui est retenue dans la version définitive.

D'abord, le $\mathrm{f}^{\circ} 530$ met en place une opposition forte entre les deux personnages: Pécuchet, soupçonneux, découvre des fautes historiques dans les romans de ce genre, qu'ils soient l'œuvre de Soulié, Dumas, Scott ou Vigny52; Bouvard, quant à lui, «ayant plus d'imagination» et étant «moins minutieux», «continue quelque temps Walter Scott». L'articulation des deux moments narratifs repose donc sur une différenciation psychologique des deux caractères. Parce qu'il est intérieurement différent de Pécuchet, Bouvard peut logiquement continuer à lire des romans historiques, malgré les fautes que son compagnon y a découvertes. Ensuite, le changement d'attitude de Bouvard envers Walter Scott prend du temps. Il ne s'opère qu'au sein de la lecture qu'il partage en une période «heureuse» et une période «insatisfaite». À ce stade, deux articulations s'enchaînent donc : la première repose sur une caractérisation

52. Pour des raisons de place, nous n'avons pu reproduire les différentes étapes par lesquelles passe ce paragraphe. 
différentielle des personnages; la seconde, en revanche, surgit de l'objet d'étude lui-même.

Dans le $\mathrm{f}^{\circ} 534 \mathrm{v}^{\circ}$ se produisent plusieurs phénomènes qui vont favoriser l'évolution de notre phrase liminaire. Tout d'abord, le dernier auteur de romans historiques à exciter les foudres de Pécuchet, Vigny, disparaît : le paragraphe qui le concerne est raturé et la mention de Cinq-Mars est déplacée vers l'amont. Cette correction a pour effet de rendre, pour la première fois, contiguës la critique que fait Pécuchet de Scott et la poursuite de sa lecture par Bouvard. Pour trois raisons, cette rencontre n'a pas ici de conséquence : d'abord, le processus décrit est une correction tardive; ensuite, le bloc barré concernant Vigny sépare toujours matériellement les deux séquences; enfin, les $\mathrm{f}^{\circ} 528$ et 529 vont opérer une coupure exactement entre notre paragraphe de référence et celui qui le précède.

Autre phénomène intéressant dans le $\mathrm{f}^{\circ} 534 \mathrm{v}^{\circ}, 1 \mathrm{e}$ système de motivation évolue. La différence psychologique qui existe entre Bouvard et Pécuchet n'explique plus seulement que le premier puisse continuer à lire le baronnet malgré les fautes qu'y trouve le second : elle se voit investie d'une autre tâche, celle de motiver simultanément le revirement de Bouvard qui «continue Walter Scott mais le trouve monotone», et ceci avant même que la lecture heureuse ne soit transformée par sa durée en lecture insatisfaite. L'opposition psychologique des deux personnages ne sert plus uniquement à motiver la poursuite de la lecture mais surtout le fait que Bouvard n'y relève pas les mêmes défauts que Pécuchet : chaque tempérament, d'après Flaubert, est en effet sensible à un type de «fautes » particulier. Le «centre de gravité » de la phrase est ainsi déplacé, et l'écrivain resserre sa prose en économisant une étape argumentative.

Lorsqu'il écrit le $\mathrm{f}^{\circ} 536 \mathrm{v}^{\circ}$, Flaubert le fait à la lumière de la rédaction antérieure des $\mathrm{f}^{\circ} 528$ et 529 . Or le $\mathrm{f}^{\circ} 528$ laisse apparaître, de la part de Pécuchet, une inflation des notations critiquant Walter Scott. Ce développement est repris dans le premier jet du fo 529 auquel succède la réécriture, dans le premier jet du $\mathrm{f}^{\circ} 536 \mathrm{v}^{\circ}$, de la fin du $\mathrm{f}^{\circ} 534 \mathrm{v}^{\circ}$ et du début du $\mathrm{f}^{\circ} 527 \mathrm{v}^{\circ}$. Puis Flaubert corrige le $\mathrm{f}^{\circ} 529$ et relit le $\mathrm{f}^{\circ} 536 \mathrm{v}^{\circ}$ dans la continuité. Or les exemples développés par Pécuchet dans sa critique historique de Scott sont tellement intimement liés à sa psychologie qu'ils rendent superflue et redondante la mention explicite des différences avec Bouvard ${ }^{53}$. Rendre sensible cette opposition est le but qui a guidé le choix des arguments retenus par Flaubert dans les f ${ }^{\circ} 528$ et 529 . Cette préoccupation rejoint ainsi la modification du paragraphe suivant dont nous avons noté les débuts dans le $\mathrm{f}^{\circ} 534 \mathrm{v}^{\circ}$.

La correction du $\mathrm{f}^{\circ} 536 \mathrm{v}^{\circ}$ voit donc Flaubert barrer la parenthèse psychologique concernant Bouvard : elle a servi à informer le texte et peut maintenant disparaître. L'opposition psychologique est dorénavant inscrite dans la matière même de cette phrase et des rapports qu'elle entretient avec le paragraphe qui la précède. Deux corrections vont alors être successivement tentées. La première («passa aux autres romans ») ne résiste pas à l'examen. En effet, elle revient à la motivation première : si Bouvard modifiait la nature de ses lectures, il prendrait en compte les critiques spécifiquement historiques que Pécuchet adresse à Walter Scott. Or justement, ils sont différents, et ce qui gêne Pécuchet ne doit pas gêner Bouvard. C'est ce que confirme la seconde correction en proposant la structure finalement retenue : «Bouvard n'en continua pas moins Walter Scott». L'utilisation de l'adverbe d'intensité couplé avec une négation a plusieurs effets. Elle permet tout d'abord d'insister sur l'assiduité dont fait preuve Bouvard dans la poursuite de sa lecture, et ceci malgré les critiques de Pécuchet. L'atténuation de l'intensité étant niée, cette dernière en sort d'autant renforcée. La correction présente aussi l'intérêt, pour traduire l'idée d'intensité, de réintroduire l'adverbe « moins » qui, ayant été supprimé dans la parenthèse psychologique, rappelle ainsi «quelque chose» de la comparaison initialement posée.

Ainsi, grâce à l'évolution simultanée de son contexte immédiat, la phrase initiale de notre paragraphe de référence est passée de la mention explicite d'une différence psychologique à son intériorisation dans sa structure et sa matière même. Mais le texte garde en mémoire ce constant souci de différenciation qu'il a pourtant cherché à atténuer ${ }^{54}$. L'analyse de cet exemple montre alors assez clairement que l'unité-paragraphe n'est pas une structure de base de la composition flaubertienne.

53. Seul l'esprit (dépourvu de tout lyrisme!) de Pécuchet pouvait pointer la contradiction entre la description donnée par Walter Scott du cadavre de Charles le Téméraire (son air «menaçant»), et le fait que, d'après certains, des loups avaient devancé ceux qui découvrirent ce corps... 


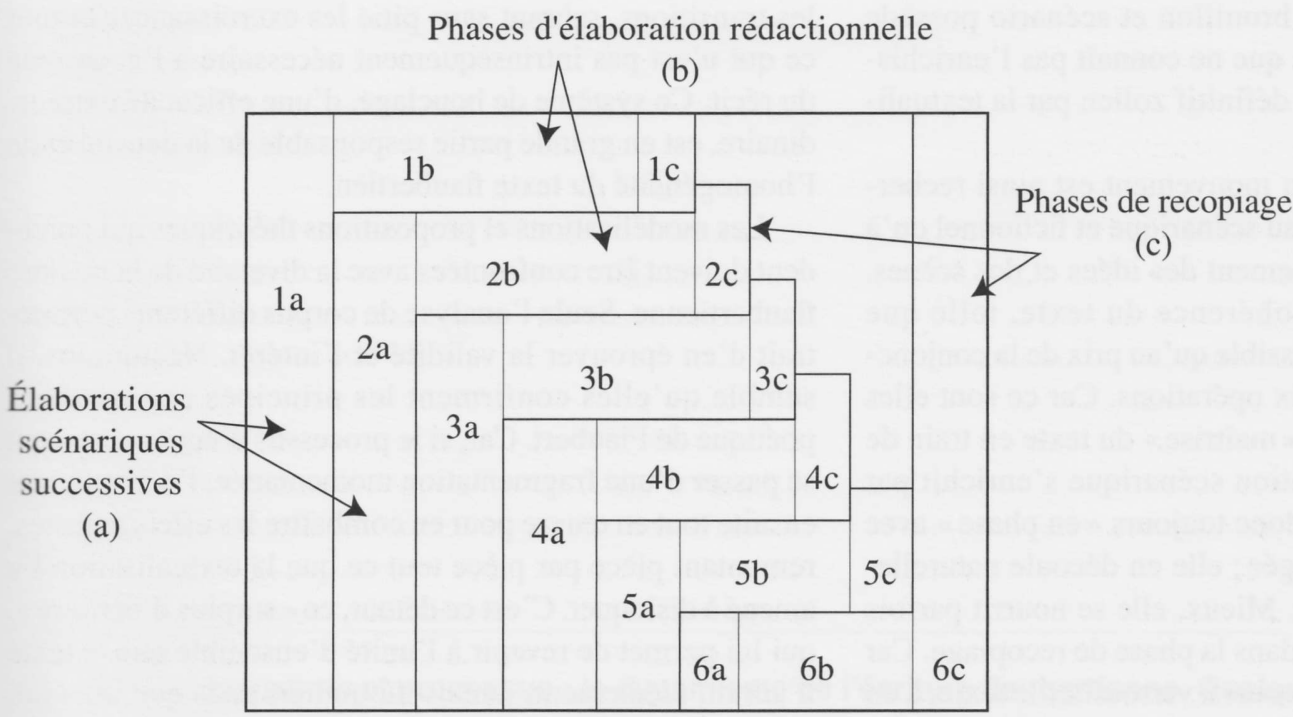

Fig. 9 :

L'écriture à programme dynamique

\section{L'écriture à programme dynamique}

Au terme de cette vue d'ensemble tant théorique que pratique, on propose de distinguer, au sein du type d'écriture à programme, une écriture à programme statique et une écriture à programme dynamique. La première conserve toutes les caractéristiques mises en place précédemment; c'est le type d'écriture de Zola. En revanche, l'écriture de Flaubert appartient à la seconde. Elle peut être représentée dans l'espace à partir d'un enchaînement de phases angulaires.

Le récit de genèse du chapitre modélisé dans la figure 9 s'obtient à partir de l'enchaînement de six phases angulaires. L'écriture concerne successivement les zones 1a, 1b, 1c, puis 2a, 2b, 2c, etc. Ainsi, on voit que Flaubert cherche constamment à faire tenir ensemble les deux dimensions de la séquence qu'il élabore, celle qui est orientée vers l'amont, et celle qui est orientée vers l'aval, non seulement au niveau microstructurel, mais aussi à l'échelle du chapitre tout entier. Chaque élaboration scénarique (a) est suivie par la rédaction d'une portion de texte (b), et chaque phase de recopiage (c) précède une réélaboration des scénarios du texte restant (a). Dans la pratique, une fois sur deux en moyenne, ce n'est pas la totalité du mouve- ment qui est réélaborée, mais une partie seulement. Grâce à ce processus, la rédaction d'un passage procède du scénario, mais simultanément, le scénario s'élabore et se complète à mesure que le texte se construit 55 . Cette inter-

54. Voir l'article cité de Daniel Ferrer, p. 103-106. Comme le précise l'auteur : «Nous avons parlé d'empreinte du contexte : il faut se souvenir que l'empreinte n'est pas la chose même. La mémoire ne saurait être absolue [...]» (p. 104). Dans notre exemple, en effet, la différenciation psychologique des deux personnages se déduit clairement de l'enchaînement des deux paragraphes tels qu'ils apparaissent dans la version définitive. Il n'en reste pas moins qu'elle peut donner lieu à un déplacement interprétatif : non plus une opposition entre deux critiques d'un même auteur issues de deux tempéraments différents, mais une opposition entre une conclusion critique et un entêtement stupide...

55. Voir l'examen complet que donne Pierre-Marc de Biasi des différentes implications de cette interaction : «What is a literary draft? Toward a functional typology of genetic documentation », Yale French Studies, 89 «Drafts », p. 26-58; et particulièrement p. 48 : «Scriptability falls, in principle, more under the juridiction of a microgenetic approach, while structuring comes under a macrogenetic heading. It goes without saying, however, that these two concepts, useful for discerning and specifying quite different writing operations, are totally interdependant throughout the writer's work, as he or she shuttles constantly between the two, performing reciprocal and successive reworking operations, only interpretable when seen as interactive. The structure modifies in response to textualization as much as textualisation alters under the constraints of restructurating. » 
action flaubertienne entre brouillon et scénario possède une dimension méthodique que ne connaît pas l'enrichissement sporadique du plan définitif zolien par la textualisation qui le précède.

L'extrême cohérence du mouvement est ainsi recherchée et atteinte tant au niveau scénarique et fictionnel qu'à celui du style, de l'enchaînement des idées et des scènes. Ou plus exactement, la cohérence du texte, telle que Flaubert la conçoit, n'est possible qu'au prix de la conjonction permanente de ces deux opérations. Car ce sont elles qui donnent à l'écrivain la «maîtrise» du texte en train de se faire. En aval, l'élaboration scénarique s'enrichit par paliers successifs. Elle est donc toujours «en phase» avec la partie textuelle déjà rédigée; elle en découle naturellement et fait corps avec elle. Mieux, elle se nourrit parfois de ce que Flaubert émonde dans la phase de recopiage. Car en amont, ce recopiage concourt à verrouiller le texte, à en contrôler méthodiquement la progression, perfectionnant les transitions, sabrant sans pitié les excroissances et tout ce qui n'est pas intrinsèquement nécessaire à l'économie du récit. Ce système de bouclage, d'une efficacité extraordinaire, est en grande partie responsable de la densité et de l'homogénéité du texte flaubertien.

Les modélisations et propositions théoriques qui précèdent doivent être confrontées avec la diversité de la matière flaubertienne. Seule l'analyse de corpus différents permettrait d'en éprouver la validité et l'intérêt. Néanmoins, il semble qu'elles confirment les principes connus de la poétique de Flaubert. Car, si le processus d'écriture ne peut se passer d'une fragmentation momentanée, l'écrivain met ensuite tout en œuvre pour en combattre les effets néfastes, remontant pièce par pièce tout ce que la textualisation l'a amené à disloquer. C'est ce détour, ce «surplus d'écriture», qui lui permet de revenir à l'unité d'ensemble que le texte n'aurait, idéalement, jamais dû quitter, mais que sans lui, il n'aurait jamais pu atteindre. 
STÉPHANIE DORD-CrousLÉ enseigne la littérature française à l'université Lyon II en tant qu'ATER. Elle a soutenu sa thèse de doctorat en décembre 1998, «Bouvard et Pécuchet et la littérature. Étude génétique et critique du chapityre V de Bouvard et Pécuchet de Gustave Flaubert». Elle vient de publier une édition de Bouvard et Pécuchet chez Flammarion dans la collection «GF».

Stéphanie Dord-Crouslé, université Lyon II

Département des Lettres

18, quai Claude Bernard 69007 Lyon

\section{Entre programme et processus : le dynamisme de l'écriture flaubertienne. Quelques points de méthode}

Partant de l'opposition structurelle existant entre deux grands régimes d'écriture ( «écriture à programme » et «écriture à processus »), on cherche à caractériser le rythme particulier de l'écriture flaubertienne au sein des brouillons. Or l'analyse de cette strate génétique révèle l'existence de moments ternaires récurrents - ou «phases angulaires »- dont chacun constitue un ensemble clos et cohérent, articulant avec régularité les axes paradigmatique et syntagmatique, d'abord dans une phase prospective d'élaboration scénarique, puis au sein d'une élaboration rédactionnelle plus restreinte, et enfin dans un moment de « recopiage » comprenant une dimension rétrospective. La mise au jour des principes et des conséquences de cette alternance interactive permet alors de définir spécifiquement l'écriture de Flaubert comme une «écriture à programme dynamique».

Partiendo de la oposición estructural que existe entre dos grandes regímenes de escritura («escritura con programa» y «escritura con proceso »), se intenta caracterizar aquí el ritmo particular de la escritura flaubertiana en el seno de los borradores. Ahora bien, el análisis de este estrato genético revela la existencia de momentos ternarios recurrentes -0 « fases angulares » - , cada uno de los cuales constituye un conjunto cerrado y coherente, que articula con regularidad los ejes paradigmático y sintagmático, ante todo en una frase prospectiva de elaboración guionística; luego, en el seno de una elaboración redaccional más restringida,y, finalmente, en un momento de «re-copiado », que comprende una dimensión retrospectiva. El estudio de los principios y de las consecuencias de esta alternancia interactiva permite entonces definir específicamente la escritura de Flaubert como una « escritura con programa dinámico».
Vom strukturellen Gegensatz der beiden Grundschreibweisen ausgehend, wird versucht, den Flauberteigenen Rhythmus der Brouillons zu charakterisieren. So erweist sich diese genetische Schicht als reich an Dreiermomenten oder sogenannten «Winkelphasen », deren jede einzeln ein zusammenhängendes und abgeschlossenes Ganzes bildet, welches die paradigmatischen und syntaktischen Achsen regelmäßig antreibt. Dies zunächst in einer prospektiven Phase des szenarischen Erarbeitens, dann innerhalb einer engeren abfassenden Bearbeitung und schließlich in einem Moment des Abschreibens, das eine rückwärtsgerichtete Dimension enthält. Das Aufzeigen dieser Prinzipien und der Folgen dieses interaktiven Wechselspiels ermöglicht die Spezifizierung der Schreibweise von Flaubert als ein «dynamischproduktorientiertes Schreiben ».

Prendendo le mosse dall'opposizione strutturale esistente fra due grandi tipi di scrittura («scrittura a programma» e «scrittura a processo »), si cerca di caratterizzare il ritmo particolare della scrittura flaubertiana all'interno degli scartafacci. L'analisi di questo strato genetico rivela l'esistenza di momenti ternari ricorrenti - o di «fasi angolari »-di cui ognuno costituisce un insieme chiuso e coerente, articolando con regolarità gli assi paradigmatici e sintattici, dapprima in una fase prospettica di elaborazione degli scenari del romanzo, poi all'interno di una elaborazione redazionale più ristretta, e infine in una fase di «ricopiatura » che comprende una dimensione retrospettiva. Evidenziare i principi e le conseguenze di questa alternanza interattiva permette allora di definire specificamente la scrittura di Flaubert come une «scrittura a programma dinamique». 